\title{
Upregulated IncARAT in Schwann cells promotes axonal regeneration through recruiting macrophages and inducing macrophages M2 polarization
}

\section{Gang Yin}

Shanghai General Hospital

Yaofa Lin

Shanghai General Hospital

\section{Peilin Wang}

Shanghai General Hospital

Jun Zhou

Shanghai General Hospital

Haodong Lin ( $\square$ haodonglin@hotmail.com )

Shanghai General Hospital https://orcid.org/0000-0003-3461-3430

\section{Research Article}

Keywords: Schwann cells, Macrophages, Exosome, LncARAT, Peripheral nerve repair.

Posted Date: October 11th, 2021

DOI: https://doi.org/10.21203/rs.3.rs-543511/v2

License: (c) (i) This work is licensed under a Creative Commons Attribution 4.0 International License. Read Full License 


\section{Abstract}

\section{Background}

Axonal regeneration following peripheral nerve injury largely depends on a favorable microenvironment. Schwann cells (SCs) play a crucial role in axonal regeneration by interacting with macrophages, but the mechanisms underlying macrophages recruitment and polarization remain unclear.

\section{Methods}

The total RNA of crushed sciatic nerves and intact contralateral nerves was extracted and used to RNAsequencing (RNA-seq). The differentially expressed long noncoding RNA (IncRNA) and mRNAs were analyzed using bioinformatics analysis, and were verified using qPCR and western blot analysis. The putative role of IncRNA in nerve regeneration was analyzed in vitro and in vivo. Macrophage polarization phenotype was identified by assessing IL-10, Arg-1, and CD206.

\section{Results}

Here we identified an IncRNA, termed Axon Regeneration-Associated Transcript (IncARAT), upregulated in SCs and SCs-derived exosomes after crushed sciatic nerves (CSN). LncARAT contributed to axonal regeneration and improved motor functional recovery. Mechanistically, IncARAT epigenetically activated CCL2 expression by recruiting KMT2A to CCL2 promoter, which resulted in an increased H3K4 trimethylation and CCL2 transcription in SCs. CCL2 upregulation facilitated the infiltration of macrophages into the injured nerves. Meanwhile, IncARAT-enriched exosomes were released from SCs and incorporated into macrophages. Once in macrophage, IncARAT functioned as an endogenous sponge to adsorb miRNA-329-5p, resulting in an increased SOCS2 expression, which facilitated macrophage M2 polarization through a STAT1/6-dependent pathway, thus promoted axonal regeneration.

Conclusions

LncARAT may serve as a promising therapeutic avenue for peripheral nerve repair.

\section{Introduction}

Unlike the central nervous system (CNS), the peripheral nervous system (PNS) has a higher regenerative abilities following mechanical injury, toxic or inflammatory responses $(1,2)$. The PNS possesses intrinsic regenerative capacity mainly because of Schwann cells (SCs), a glia cell, which can form and maintain the myelin sheath around axons to support axonal regeneration $(3,4)$. Successful regeneration depends on both neurons and non-neuronal cells such as SCs and immune cells (5). The inflammatory responses are mainly fulfilled by macrophages in response to PNI. Macrophages are highly heterogeneous, and can be polarized to pro-inflammatory phenotype (M1) and anti-inflammatory phenotype (M2) by their microenvironment $(6,7)$. Circulating macrophages are attracted to lesion site after injury, whereby 
macrophages not only exert a crucial role in engulfing the inhibitory regeneration signals from myelin debris, but also polarize to anti-inflammatory phenotype (M2 macrophages) to facilitate axon regeneration (8-10).

Emerging studies have demonstrated that the interactions of SCs with macrophages exert a crucial role in Wallerian degeneration and axonal regeneration after PNI (11). Firstly, SCs contribute to macrophage infiltration by secreting varied types of ligands including cytokines and chemokines. SCs-derived Gelsolin, an actin filament severing protein, is essential to macrophages motility in vitro and macrophages recruitment to injury site in vivo (12). SCs-secreted periostin facilitates macrophages infiltration and results in an autoimmune peripheral polyneuropathy (13). Several chemokines and cytokines, such as (CC motif) ligand 2 (CCL2), IL-1a, IL-1 $\beta$ and TNF-a, are also produced by SCs under the stimulation of degenerated neural tissues to promote macrophages recruitment (14-17). Secondly, macrophages regulate the maturation of SCs after PNI. Growth arrest-specific 6 (Gas6) is expressed by macrophages to regulate SCs function, and loss of Gas6 in macrophages causes an abnormal SCs response and impairs SCs remyelination (11). Microvesicles derived from M2 macrophages contribute to the proliferation and migration of SCs (18). Thirdly, SCs promote macrophage M2 polarization after PNI. Co-culture of monocytic cells with SCs induced M2 phenotypic polarization as evidenced by upregulated expression of M2 marker CD163 (19). Stratton et al., demonstrated that although SCs do not produce classic M2associated cytokines, SCs can potently induce macrophage M2 polarization (20). The underlying mechanisms that SCs regulate macrophage polarization remain poorly understood.

Exosomes play an important role in cell-cell communication via transferring biological cargo including protein, RNA and DNA among different cells (2). Recent studies have demonstrated that SCs-derived exosomes support axonal regeneration (2). Furthermore, modification of exosomal miRNA profile facilitates neurite growth following nerve injury (21). Long noncoding RNAs (IncRNAs), a large class of non-protein-coding transcripts that are over 200nt in length, have emerged as key regulators of axonal regeneration and functional recovery after nerve injury $(22,23)$. However, the regulatory roles of the IncRNA/miRNA/mRNA axis in these processes remain unclear. In the present study we identified a novel IncRNA NONRATT026656.2, termed Axon regeneration-associated transcript (IncARAT), which was upregulated in SCs. Upregulated IncARAT in SCs promoted CCL2 expression to recruit macrophages to injured nerves. Furthermore, IncARAT was also transferred into infiltrating macrophages via SCs-derived exosomes and promoted macrophage M2 polarization to support axonal regeneration.

\section{Materials And Methods}

\subsection{Rat model of crushed sciatic nerves (CSN)}

All experimental protocols of animal model were approved by the Ethics Committee of the Shanghai General Hospital, Shanghai JiaoTong University School of Medicine. Healthy Sprague Dawley (SD) rats (180-220g) were obtained from the Zhejiang Chinese Medical University Laboratory Animal Research Center (Zhejiang, China) and maintained in 12-h-light/12-h-dark conditions with ad libitum access to food 
and water. Every effort was made to minimize the number of animals used and their suffering. The rat model of CSN was carried out according to the previous description (24). In brief, all rats were anesthetized with $1 \%$ pentobarbital sodium $(40 \mathrm{mg} / \mathrm{kg})$, fixed on the operating table and disinfected with $1 \%$ iodophor solution. The upper right femoral posterior incision was taken, skin and subcutaneous fascia were incised layer by layer, and the sciatic nerve was fully exposed. At a distance of $0.5 \mathrm{~cm}$ from its bifurcation, the nerve was compressed with a pair of 14-cm hemostat forceps for $30 \mathrm{sec}$.

\subsection{RNA-sequencing}

Total RNA was extracted from the distal stumps of CSN $(0.5 \mathrm{~cm})$ and intact contralateral nerves at 4 dpi as described previously (25). In brief, RNA was treated with amplification-grade DNase I (Invitrogen, Carlsbad, CA, USA). To quantify circRNAs, Epicentre RNaseR (Lucigen, Middleton, WI, USA) was applied to degrade linear RNAs and subsequently purified the resulting using a RNeasy MinElute Cleanup Kit (Qiagen, Duesseldorf, Germany). Total RNA of each sample was quantified using the NanoDrop 1000 Spectrophotometer (Nanodrop, Waltham, MA, USA). The total RNA samples ( $3 \mathrm{mg}$ ) were treated with the RiboMinus Eukaryote Kit (Invitrogen) to remove rRNA. cDNA libraries were generated as the lllumina TruSeq RNA Sequencing (RNA-Seq) protocol and sequenced on an Illumina HiSeq 2000 sequencing platform.

\subsection{Electrophysiological Assessment}

At 28 days post-injury (dpi), rats were subjected to an electrophysiological test according to previously described protocols $(n=5)(26)$. In brief, the sciatic nerve near the repair site was re-exposed, a pair of stimulating electrodes ( $13 \mathrm{~mm}$ long, $0.5 \mathrm{~mm}$ in diameter) was inserted $3 \mathrm{~mm}$ near the crushed site to stimulate the sciatic nerve, and a pair of needle electrodes was inserted subcutaneously into the middle of the intrinsic foot muscle to record the compound muscle action potential (CMAP) using EMG Evoked Potentiometer (MEB-9200K, Nihon Kohden, Japan). The amplitude and latency of each test were analyzed to determine the nerve conduction intensity and nerve conduction velocity, respectively.

\subsection{Walking track analysis}

To perform functional analysis of movement, we applied propylene pigments to the plantar surface of the hind paws of rats ( $28 \mathrm{dpi}$ ) and allowed them to walk along white paper-covered corridors. Footprints from ipsi- and contralateral paws were analyzed through measuring the print length $(\mathrm{PL})$ and the distance between 1 st and 5 th toes. The three parameters were combined to obtain the Sciatic Functional Index (SFI) (27), which quantifies the changes in the walking pattern ( 0 for uninjured; -100 for maximally impaired gait).

\subsection{Tissue preparation and RNA extraction}

Nerve lesions were produced on the right side and the intact contralateral nerve served as the uninjured control. At the appropriate time points, nerves were harvested and processed for histology or immediately frozen in liquid nitrogen for subsequent extraction of RNA.

\subsection{Primary SCs culture}


SCs primary cultures were obtained from newborn SD rat sciatic nerves and the fibroblasts were removed with fibronectin-specific antibody and rabbit complement (Thermo Fisher Scientific, Waltham, MA, USA) according to the method previously described (28). Primary culture of SCs was maintained in DMEM containing $10 \% \mathrm{FBS}$ at $37^{\circ} \mathrm{C}$ under humidified $5 \% \mathrm{CO}_{2}$. SCs growth was observed under an inverted phase contrast microscope (Thermo Fisher Scientific). SCs cultures were passaged no more than 5 times before conducting experiments. SCs $\left(1 \times 10^{5}\right)$ and macrophages $\left(1 \times 10^{5}\right)$ were co-cultured in DMEM medium containing 10\% FBS using transwell inserts (BD Biosciences, CA, USA).

\subsection{Isolation of SCs-derived exosomes (SCs-Exo) and identification}

The exosomes were isolated from SCs using Exoquick Reagent (SBI) as the manufacturer's instruction. Briefly, conditioned media were incubated with Exoquick reagent (5:1) for over $12 \mathrm{~h}$, centrifuged at 1,500 g for $30 \mathrm{~min}$ and pelleted exosomes were resuspended in $100 \mathrm{~mL}$ PBS.

Transmission electron microscopy (TEM) was used for morphological observation. The exosome samples were prepared as described above. For TEM, briefly, the exosomes were fixed with $2.5 \%$ glutaraldehyde overnight at $4^{\circ} \mathrm{C}$. The solution was centrifuged at $100,000 \times g$ to remove the glutaraldehyde, and the exosomes were washed three times with PBS. Then, the exosomes were stained with $3 \%$ phosphotungstic acid aqueous solution and fixed on copper mesh formvar grids. A transmission electron microscope (JEM-1010; JEOL, Tokyo, Japan) was used to detect the exosomes. Moreover, to analyze the distribution of particle size of the exosomes, a partial sample of exosomes was added to the sample cell without dilution. All operations were conducted in accordance with the instruction manual of

the ZetaView ${ }^{\circledR}$ NTA technique (Particle Metrix, Germany). $10 \mu \mathrm{g}$ of exosomes (re-suspended in PBS) were used to treat macrophages according to previous reports (2) and our preliminary results.

\subsection{Exosome labeling and tracking}

Exosomes were isolated from the culture medium and labeled with PKH67 Green Fluorescent membrane linker dye (Sigma-Aldrich, St. Louis, MO, USA) according to manufacturer's instructions. Then, the labeled exosome pellets were resuspended and added to the unstained macrophages for exosomes uptake studies. After incubation for $30 \mathrm{~min}, 2 \mathrm{~h}$, or $12 \mathrm{~h}$ at $37^{\circ} \mathrm{C}$, cells were observed by fluorescence microscopy.

\subsection{Immunofluorescence}

Rat sciatic nerves were fixed in situ with 4\% PFA for 10 min, dissected, embedded in O.C.T. Compound (Tissue Freezing Medium; Solarbio, Shanghai, China), and frozen at $-80^{\circ} \mathrm{C}$. Sciatic nerve cryosections (5$\mu \mathrm{m}$ thick) were first incubated with acetone for $10 \mathrm{~min}$ at $-20^{\circ} \mathrm{C}$, washed in PBS $/ 0.1 \%$ Tween 20 , blocked for $30 \mathrm{~min}$ at room temperature (RT) in blocking buffer $(0.3 \%$ Triton X-100/10\% Goat serum/phosphate buffer saline $1 / 4 \mathrm{PBS}$ ), and incubated with primary antibodies overnight at $4{ }^{\circ} \mathrm{C}$ in blocking buffer. Cryosections were first incubated with 70\% Ethanol for 5 min at RT, washed with PBS and incubated for $40 \mathrm{~s}$ with $40 \mathrm{mg} / \mathrm{ml}$ Proteinase $\mathrm{K}$, before incubation with blocking buffer. Sections were then washed 3 
times in blocking buffer and secondary antibodies were incubated for $1 \mathrm{~h}$ at RT in the dark. Sections were washed again, incubated with DAPI for 5 min at RT, washed and mounted in Citifluor (Agar Scientific).

Primary antibodies used for Immunofluorescence were as follow: Neurofilament (1:1000, Abcam, ab8135), SCG10 (1:500, Abcam, ab115513), IBA1 (1:100, Abcam, ab178847), NF200 (1:100, Abcam, ab82259), CD68 (1:100, Abcam, ab125212). All secondary antibodies were also purchased from Abcam. Photos were acquired using a Leica TCS SP-II confocal microscope.

\subsection{0 (Fluorescence in situ hybridization) FISH}

The subcellular localization of IncARAT was assessed using FISH assay with RiboTM IncRNA FISH Probe Mix (Green) (RiboBio, Guangzhou, China). The sciatic nerve tissue sections were fixed with $4 \%$ Paraformaldehyde (PFA). Slides were pretreated with protease $K(2 \mu \mathrm{g} / \mathrm{mL})$, glycine and acetic anhydride, followed by pre-hybridization for $1 \mathrm{~h}$ and hybridization at $42^{\circ} \mathrm{C}$ with probes $(250 \mu \mathrm{L}, 300 \mathrm{ng} / \mathrm{mL})$ against IncARAT. Finally, slides were stained with phosphate buffered saline with DAPI (Sigma-Aldrich). Finally, 5 random fields acquired from each slide were observed and photographed by a fluorescence microscope.

\subsection{Semithin and ultrathin sections and electron microscopy}

After killed with $30 \mathrm{mg} / \mathrm{kg} 3 \%$ pentobarbital sodium i.p., the rat' sciatic nerves were fixed in situ with $3 \%$ paraformaldehyde and $0.15 \%$ glutaraldehyde in $0.1 \mathrm{M}$ phosphate buffer, $\mathrm{pH} 7.4$. Fixed tissues were postfixed in $2 \%$ osmium tetroxide, dehydrated through a graded acetone series as described previously, and embedded in Spurr's resin (Electron Microscopy Sciences, EMS). Semithin sections were stained with 1\% Toluidine blue for analysis at the light microscope, and ultrathin sections (70-nm thick) were made. All analyses were done at $5 \mathrm{~mm}$ distal to the lesion site. No contrasting reagent was applied. Images were acquired using a Philips CM 100 BIOTWIN equipped with a Morada sidemounted digital camera (Olympus).

\subsection{RNA pull-down and RNA immunoprecipitation (RIP)}

RNA pull-down assays were performed with the Magnetic RNA-Protein Pull down Kit (Thermo Scientific) according to the manufacturer's instructions. $3 \mu \mathrm{g}$ biotin-labeled RNA and $1 \mathrm{mg}$ of extract were used in each pull down assay. The retrieved protein was separated on polyacrylamide gel electrophoresis (PAGE) gels and visualized by standard immunoblotting.

RIP assay was performed using the EZ-Magna RIP kit (Millipore, MA, USA). In brief, $1 \times 10^{7}$ cells were harvested and lysed with RIP lysis buffer with one freeze-thaw cycle. Cell extracts were coimmunoprecipitated using anti-KMT2A (ab272023), anti-KMT2B (ab104444), anti-KMT2D (ab224156) or Ago2 (ab226943) antibody, and the retrieved RNA was subjected to qPCR analysis.

\subsection{ChIP and ChIRP analysis}


The ChIP experiments were performed using the ChIP kit (Millipore, MA, USA). A total of $1 \times 10^{6}$ cells were fixed in $1 \%$ formaldehyde at room temperature for $10 \mathrm{~min}$, and the nuclei were isolated with nuclear lysis buffer supplemented with a protease inhibitor. The chromatin DNA was sonicated and sheared to lengths between 100 and $200 \mathrm{bp}$. The sheared chromatin was immunoprecipitated at $4^{\circ} \mathrm{C}$ overnight using an antiIncARAT antibody, anti-KMT2A antibody or anti-H3K4me3 antibody (Abcam, MA, USA). Normal mouse IgG was used as the NC. The ChIP-qPCR primers are listed in Supporting Table S9.

The Magna ChIRP RNA Interactome Kit was purchased from Millipore (Millipore, MA, USA) and used according to the manufacturer's instructions. In brief, the probes were designed using a single-molecule FISH online designer, were biotin-labeled at the 3'end and were divided into an "odds" or an "even" groups. A total of $2 \times 10^{7}$ cells were cross-linked for each hybridization reaction. Then, the cell lysate was sonicated to shear the chromatin into 100-200 bp fragments. The sonicated cell lysates were hybridized with a mixture of biotinylated DNA probes for $4 \mathrm{~h}$ at $37^{\circ} \mathrm{C}$. Then, the binding complexes were recovered using streptavidin-conjugated magnetic beads. Finally, DNA, RNA and protein were eluted and purified from the beads. The probes used in the ChIRP assay are listed in Supporting Table S9.

\subsection{Lentivirus construction and RNA interference (RNAi)}

The Lentivirus harboring IncARAT (Lv-IncARAT) or SOCS2 (Lv-SOCS2) CDNA was produced by GenePharma (Shanghai, China). An unrelated shRNA without any match with the rat genomic sequence was used as a control (Lv-Cont). Small interference RNA (siRNAs) for specifically inhibiting IncARAT (siIncARATs), SOCS2 (si-SOCS2s), or short hairpin RNA (shRNAs) for specifically inhibiting IncARAT (shIncARATs) was designed and produced by GenePharma (Shanghai, China), and transfected with HiPerFect Transfection Reagent (Qiagen, CA, USA). The siRNA and shRNA sequences were showed in Supporting Table S9.

\subsection{Reverse transcription-quantitative PCR (qPCR)}

Total RNA from tissues and cells was extracted using a TRIzol® kit (Invitrogen, Carlsbad, CA, USA) following the manufacturer's instruction. Reverse transcription was carried out using a PrimeScript RT reagent Kit (Takara Bio, Inc., Tokyo, Japan). The RT system of $10 \mu \mathrm{L}$ were carried out according to the manufacturer's instruction. RT conditions were $37^{\circ} \mathrm{C}$ for $15 \mathrm{~min}$ and $85^{\circ} \mathrm{C}$ for $5 \mathrm{~s}$. mRNA, IncRNA and miRNA expression levels were determined using the SYBR Green Supermix (Invitrogen) on the Applied Biosystems 7300 real-time PCR system. Thermocycling conditions were $95^{\circ} \mathrm{C}$ for $10 \mathrm{~min}$, following by 35 cycles of $95^{\circ} \mathrm{C}$ for $10 \mathrm{~s}, 58^{\circ} \mathrm{C}$ for $15 \mathrm{~s}$ and $72{ }^{\circ} \mathrm{C}$ for $20 \mathrm{~s}$, and final $72{ }^{\circ} \mathrm{C}$ for $20 \mathrm{~min}$. $\beta$-actin was used as the internal control. All qPCR experiments were performed at $\geq 3$ times, and the primer sequences were shown in Supporting Table S9.

\subsection{Western blot analysis}

Total protein was extracted from nerves or macrophages using RIPA buffer (Solarbio). The concentrations of the extracted nuclear and cytoplasmic fractions were quantified using a BCA protein assay kit (Pierce). A total of $50 \mu \mathrm{g}$ protein per sample was separated by SDS-PAGE $(10 \%)$ and then 
transferred to a PVDF membrane, prior to blocking with $5 \%$ non-fat milk in $1 \mathrm{xTBST}$, overnight at $4^{\circ} \mathrm{C}$. The membrane was then incubated with anti-CCL2 (1:2000; ab25124; Abcam, Cambridge, MA, USA), anti-iNOS (1:500; ab15323; Abcam), anti-Arg1 (1:1000; ab91279; Abcam), anti-CD206 (1:1000; ab125028; Abcam), anti-GAPDH (1:10000; ab181602; Abcam), anti-SOCS2 (1:1000; PA5-17219; Thermo Fisher Scientific) and Actin (1:5000; ab179467; Abcam) primary antibodies overnight at $4{ }^{\circ} \mathrm{C}$. After washing 3 times in 1xTBST, the membranes were incubated with the corresponding HRP-conjugated secondary antibody (1:5000; ab205718; Abcam) for $1 \mathrm{~h}$ at room temperature. The immunoreactive proteins were visualized by an enhanced chemiluminescence reaction, and the band density was calculated by ChemiDoc $^{\mathrm{TM}} \mathrm{XRS}+$ Imaging system (Bio-Rad).

\subsection{Dual-luciferase reporter assay}

Recombinant plasmids of pGL3-IncARAT-Wt, pGL3-IncARAT-Mut, pGL3-SOCS2-3'UTR-Wt, and pGL3-

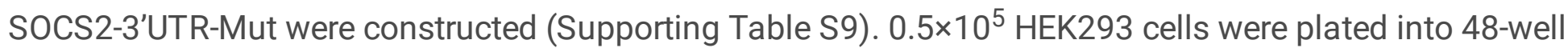
plate and co-transfected with $50 \mathrm{nM}$ of miRNA-329-5p (or miRNA control), $20 \mathrm{ng}$ of either pGL3-IncARATWt, pGL3-IncARAT-Mut, pGL3-SOCS2-3'UTR-Wt, or pGL3-SOCS2-3'UTR-Mut, and 2 ng of pRL-TK (Promega, Madison, WI) using HiPerFect Transfection Reagent (Qiagen). pRL-TK was served as the internal control. HEK293 cells were collected and lysed $48 \mathrm{~h}$ after transfection and the luciferase activity was assessed using the Dual-Luciferase Reporter Assay System (Promega).

\subsection{Cell migration}

Cell migration was assessed using transwell chamber (BD Biosciences, 8- $\mu \mathrm{m}$ pore size, 24-well). Briefly, macrophages $\left(1 \times 10^{4}\right)$ suspended in $200 \mathrm{~mL}$ serum-free medium were seeded into the upper chamber, and $1 \times 10^{4}$ SCs in $800 \mathrm{~mL}$ medium containing $10 \%$ FBS were added to the bottom chamber. After 24 hours of culture, cells were then stained with $0.1 \%$ crystal violet for 30 minutes, and nonmigrating cells were removed. Six visual fields were randomly chosen to calculate the number of migrated cells.

\subsection{Macrophage depletion}

Macrophages were depleted through i.p. administration of clodronate liposome in rats according to previously reported method (29). In brief, $0.8 \mathrm{ml}$ clodronate liposome $(7 \mathrm{mg} / \mathrm{ml}$ ) (Clodrosome, Encapsula NanoSciences, USA) was injected into rats with CSN. Control rats received i.p. administration of equal volume of PBS liposome.

\subsection{Statistical analysis}

All data were presented as the mean \pm standard deviation (SD). The significance of differences between groups were assessed by Student's $t$ test and multiple group comparisons were performed using one-way ANOVA followed by the Scheffé test. SPSS 20.0 statistical software was applied for statistical analyses. $p<0.05$ was statistically significant.

\section{Results}




\subsection{LncRNA expression profile in crushed sciatic nerves}

To investigate the potential function of IncRNAs in response to sciatic nerve damage and repair, total RNA was acquired from the distal stumps of crushed sciatic nerves and intact contralateral nerves for RNAsequencing (RNA-seq). A total of 98 differentially expressed IncRNAs (including 46 up-regulated and 52 down-regulated IncRNAs, Supporting Table S1) and 77 differentially expressed mRNAs (including 55 upregulated and 22 down-regulated mRNAs, Supporting Table S2) were identified between crushed sciatic nerves and normal control (Fig. $1 \mathrm{~A}, \log 2 \mathrm{FC}>1$ and $\mathrm{p}<0.001$ ). Functional enrichment analysis by Gene Ontology (GO) revealed that differentially expressed mRNAs were enriched in $362 \mathrm{GO}$ biological process terms, and these terms were mainly associated with immune and migration, including cytokine activity and regulation of leukocyte migration (Supporting Figure S1A and Supporting Table S3). Besides, differentially expressed mRNAs were significantly enriched in 86 KEGG pathways, and these pathways were mainly related to cell growth, cell death, and nervous system (Supporting Figure S1B and Supporting Table S4). A Venn diagram analysis identified 4 overlapping IncRNAs ranked by $p$ values and fold changes (Supporting Figure S1C and Supporting Table S5-7). Here we focused on NONRATT026656.2 (IncRNA-Axon regeneration-associated transcript, thereafter named IncARAT) and investigated its biological role in axonal regeneration.

The full-length sequence of IncARAT was identified using rapid amplification of cDNA ends (RACE, Supporting Figure S1D). Expected size of IncARAT was ascertained using northern blot analysis (Supporting Figure S1E). Blast analysis against the NCBI database showed that rat IncARAT is located on chromosome 7, and human IncARAT is located on chromosome 12. Rat and human IncARAT overlaps on the sense strand with coding gene Gas2l3 (growth arrest specific 2 like 3 ). However, sequence analysis could not predict a protein of above 60 amino acids using Open Reading Frame Finder from NCBI (Supporting Figure S1F). Furthermore, we used Coding-Non-Coding Index $(\mathrm{CNCl})$, Coding Potential Calculator algorithm (CPC2), and txCdsPredict from UCSC to calculate the coding potential of IncARAT. All three algorithms predicted that IncARAT does not possess protein-coding potential (Supporting Figure S1G). Moreover, IncARAT does not contain valid Kozak consensus sequence.

The expression of IncARAT was then assessed in crushed sciatic nerves at different time points postinjury. Figure 1B showed that the IncARAT level was increased at 1 day post-injury (dpi), peaking at $7 \mathrm{dpi}$ and declining thereafter, but still remaining high at $21 \mathrm{dpi} \mathrm{compared} \mathrm{with} \mathrm{uninjured} \mathrm{nerves.} \mathrm{Moreover,}$ RNA-FISH assay showed that IncARAT level was also increased at 7 and 21 dpi (Fig. 1C and D). Bifluorescence imaging analysis verified that IncARAT mainly located in SCs (Fig. 1E). The biological function of IncARAT on nerve regeneration was investigated by injecting LV-IncARAT or Lv-shIncARAT into sciatic nerves to enhance or repress IncARAT expression, and then damaged the sciatic nerves after 4 days. Figure $1 \mathrm{~F}$ showed that the IncARAT overexpression accelerated early demyelination of injured nerves, whereas IncARAT knockdown decreased demyelination. Immunofluorescence and western blot analysis showed that the axonal regeneration marker, SCG10 was markedly enhanced after IncARAT overexpression at $3 \mathrm{dpi}$, whereas IncARAT knockdown resulted in a decreased level of SCG10 versus control nerves (Fig. 1G, Supporting Figure S1H), indicating that upregulated IncARAT contributes to 
axonal regrowth. To evaluate the role of IncARAT in motor functional recovery after sciatic nerve damage, the amplitude and latency of the compound muscle action potential (CMAP) were assessed by electrophysiology. As shown in Fig. 1H, IncARAT overexpression resulted in a significant increase in CMAP amplitude at $28 \mathrm{dpi}$, whereas CMAP amplitude was decreased when IncARAT inhibition. Moreover, IncARAT overexpression significantly decreased CMAP latency, whereas IncARAT knockdown increased CMAP latency (Fig. 1H). Walking track technique was used to further test the role of IncARAT in motor recovery. LncARAT overexpression showed a significant decrease in the sciatic functional index versus control at $28 \mathrm{dpi}$, whereas IncARAT inhibition resulted in the increase of sciatic functional index (Fig. 1I). These data demonstrate that upregulated IncARAT promotes axonal regeneration and improves motor functional recovery after sciatic nerve damage.

\subsection{LncARAT upregulated CCL2 expression in SCs}

Although IncARAT could not regulate SCs proliferation and migration (data not shown), IncARAT promoted the infiltration of macrophages into injury site (Supporting Figure S2A), indicating that upregulated IncARAT contributed to recruit macrophages into the injury site. A IncRNA-mRNA coexpression network analysis showed that IncARAT was correlated with multiple chemokines or chemokine receptors including CCL2, CCR7 and CCRL2 (Fig. 2A). Given the important role of chemokines in macrophage recruitment, the expression of CCL2, CCR7 and CCRL2 was assessed in CSN. Western blot analysis showed that CCL2 was significantly up-regulated in injured nerves, while CCR7 and CCRL2 had no significant change (Fig. 2B, Supporting Figure S2B-F). Lv-IncARAT or Lv-shlncARAT was injected into sciatic nerves to overexpress or knockdown IncARAT, and then damaged the sciatic nerves after 4 days. Figure $2 \mathrm{C}$ showed that IncARAT overexpression further enhanced CCL2 expression in SCs, whereas IncARAT knockdown reduced CCL2 expression compared with group. LncARAT overexpression in primary SCs also resulted in a significant increase of CCL2 expression (Fig. 2D, Supporting Figure S2G and H). In animals in which CCL2 or CCR2 are deleted, neither macrophage infiltration nor the conditioning lesion response occurs in dorsal root ganglia $(9,30)$, indicating that SCs facilitate macrophage infiltration, at least in part, through IncARAT-induced CCL2 expression.

\subsection{LncARAT promoted H3K4me3 at CCL2 promoter by interacting with KMT2A}

Subcellular localization analysis was performed and data shown that IncARAT was localized to the nucleus (54\%) and cytoplasm (46\%) in SCs (Fig. 2E). To reveal the molecular mechanisms by which IncARAT stimulates CCL2 upregulation, a series of CCL2-luc promoter constructs were cloned, which range from -2000 nt to +150 nt relative to the transcriptional start site. The promoter luciferase assay showed a significant increase in the transcriptional activity of the construct from -200 to $+150 \mathrm{bp}$ rather than from +1 to $+150 \mathrm{bp}$ (Fig. 2F). Furthermore, a chromatin isolation by RNA purification (ChIRP) assay, which determines the exact locations of IncRNA binding sites on the chromatin, revealed that IncARAT bound to -19 to -127 bp (referred to as CCL2-S2; Fig. 2G). 
Lots of IncRNA epigenetically regulates target gene expression by regulating histone $\mathrm{H} 3$ methylation or demethylation at lysine or arginine residue (31-33). Our analysis found a potential chromatin H3K4me3 modification on the transcription edges of CCL2 (Fig. 2H). We thus assessed the H3K4me3 level in CSN because H3K4 methylation is commonly associated with gene activation (34). As shown in Fig. 2I, H3K4me3 level was enhanced significantly in injured nerves when compared to sham control, whereas IncARAT inhibition decreased H3K4me3 level, and H3K4me3 was observed obviously in SCs. We also performed a RNA pull down assay to identify the histone methylation modification enzymes involved in H3K4me3. The results showed that IncARAT specifically interacted with KMT2A but not with KMT2B, KMT2D (Fig. 2J), or SET1A, SET1B, or SMYD3 (data not shown). Consistently, RNA Immunoprecipitation (RIP) assays using nuclear extract demonstrated that IncARAT directly interacted with KMT2A (Fig. 2K). To verify that IncARAT increased CCL2 expression by interacting with KMT2A and catalyzing H3K4me3 at CCL2 promoter, ChIP analysis was performed using KMT2A and H3K4me3, respectively. The results from ChIP assay showed that IncARAT overexpression increased KMT2A occupancy at CCL2 promoter (Fig. 2L) and increased H3K4me3 at the promoter of CCL2 (Fig. 2M). These data suggest that IncARAT up-regulates CCL2 expression through KMT2A-mediated H3K4me3.

\subsection{SCs-derived exosomes (SCs-Exo) promoted macrophage M2 polarization}

Consistent with the in vivo results, IncRNA overexpression in cultured SCs facilitated the migration of macrophages including bone marrow-derived macrophages (BMDM) (Fig. 3A and B) and U937 cells (Supporting Figure S3A and B). Given the important role of macrophage M2 polarization in nerve regeneration, and we found that M2-macrophage was significantly increased at 7-day post-injury (dpi) (Supporting Figure S3C), consistence with the expression of IncARAT, we next investigated the effect of IncARAT on regulating macrophage polarization. Immunofluorescence analysis of CD206 revealed that M2 macrophages were observed in injured nerves, whereas IncARAT knockdown decreased dramatically the amount of M2 macrophages (Fig. 3C). We next used an in vitro co-culture system to investigate the direct interaction of IncARAT-overexpressed SCs with macrophage M2 polarization. BMDM or U937 cocultured with IncARAT-overexpressed SCs showed a marked polarization to M2, as evidenced by upregulated expression of classic M2 signature markers such as IL-10, Arg1 and CD206 at mRNA (Fig. 3D and Supporting Figure S3D) and protein level (Fig. 3E).

Exosome exerts a crucial role in cell-cell communication via transferring RNA and protein among different cells (2). Given that IncARAT was upregulated in SCs after nerves injury and IncARAT overexpression in SCs facilitated macrophage M2 polarization, it is essential to investigate whether IncARAT was transferred from SCs to macrophages to induce macrophage M2 polarization via SCs-Exo. To this end, IncARAT was overexpressed in SCs, and then the corresponding exosome (SCs-Exo/Lv-IncARAT) was collected from the conditioned media (CM) and identified by TEM, NanoSight and western blot analysis. The results from TEM showed that the collected products from SCs overexpressed with Lv-Cont or LVIncARAT had a distinctive cup shape and showed a typical lipid bilayer nanoparticle (Fig. 3F). NanoSight analysis showed that the diameter of collected products was within range of $80-130 \mathrm{~nm}$ (Fig. 3G). The

Page $11 / 30$ 
expression of exosomal markers CD9, CD81 and CD63 was also verified through western blot analysis (Fig. $3 \mathrm{H}$ ), but the absence of GM130, the cis-Golgi compartment-specific marker (data not shown). As expected, the expression level of IncARAT in exosome derived from IncARAT-overexpressed SCs (SCsExo/Lv-IncARAT) was markedly upregulated compared with SCs-Exo/Lv-Cont (Fig. 3I). Functionally, although SCs-Exo/Lv-IncARAT did not affect the migration of macrophages (Supporting Figure S3E and F), macrophages treated with SCs-Exo/Lv-IncARAT showed a marked polarization to M2 (Fig. 3J-L and Supporting Figure S3G) and protein level (Fig. 3M and N). Treatment of macrophages with GW4869, an exosome inhibitor $(35,36)$, repressed markedly SCs-Exo/Lv-IncARAT-induced macrophage M2 polarization (Fig. 30). Taken together, these results suggest that SCs facilitate macrophage migration via secreting CCL2 and then induce macrophage M2 polarization via exosomes-mediating SCs-macrophages communication.

\subsection{LncARAT was transferred from SCs to macrophages via SCs-Exo}

To verify that IncARAT was transferred from SCs to macrophages via SCs-Exo, we first assessed whether IncARAT was packed within SCs-Exo to resist RNase-mediated degradation. Figure 4A showed that the IncARAT level in SCs-Exo was kept unchanged after RNase A treatment, while additional treatment with Triton X-100 resulted in a significant decrease of IncARAT level, indicating that IncARAT was packed within exosome. We then investigated whether IncARAT could be transferred from SCs to macrophages. SCs-Exo was labeled using PKH67 and then the PKH67-SCs-Exo was used to treat macrophages. As shown in Fig. 4B, green fluorescence signals were observed in macrophages, indicating that SCs-Exos were internalized by macrophages. Forced expression of IncARAT in SCs resulted in a significant enhancement of IncARAT expression in SCs and SCs-Exo (Fig. 4C and D). Importantly, IncARAT level in recipient macrophages was increased after treatment with SCs-Exo/Lv-IncARAT compard with SCsExo/Lv-Cont (Fig. 4E). LncARAT was labeled with fluorescein amidite (FAM) and SCs-Exo was acquired after FAM-IncARAT transfection to treat macrophages. Figure 4F showed that the FAM-IncARAT was observed in macrophages, indicating that IncARAT was packed within SCs-Exos and transferred to macrophages.

We then investigated whether SCs-Exo promoted macrophage M2 polarization by delivering IncARAT. BMDM or U937 cells were treated with Lv-IncARAT and then the macrophage polarization was assayed. As shown in Fig. 4G-I and Supporting Figure S4A, forced expression of IncARAT enhanced the mRNA level of macrophage M2 markers compared with blank and LV-cont. Western blot analysis also verified that the protein levels of macrophage M2 markers were also significantly upregulated after IncARAT overexpression (Fig. 4J and K). In vivo, IncARAT overexpression also enhanced Arg1 expression, whereas IncARAT knockdown reduced Arg1 expression compared with group (Fig. 4L). These results suggest that SCs-Exo promotes macrophage M2 polarization by transferring IncARAT to macrophages.

\subsection{LncARAT functioned as a ceRNA and sponged miRNA- 329-5p to facilitate macrophage M2 polarization}


LncRNAs could act as ceRNA to regulate gene expression via sponging miRNAs in physiopathologic conditions (37). The results from subcellular localization analysis showed that IncARAT was largely located in cytoplasm in macrophages (Fig. 5A), indicating that IncARAT might act as a ceRNA. The bioinformatics tool (miRDB, http://mirdb.org/) was used to predict miRNAs that potentially interacted with IncARAT and 36 miRNAs had complementary sequence to IncARAT (Supporting Table S8). We carried out a RNA pull-down assay using biotinylated miRNA to identify the miRNAs bound to IncARAT. Figure 5B and $C$ showed that IncARAT was more enriched in miRNA-329-5p than in other miRNAs, whereas the miRNA-329-5p mutant in IncARAT binding site lost its capability bound to IncARAT in macrophages (Supporting Figure S4B). Moreover, RNA pull-down assay using biotinylated IncARAT also verified the direct combination of miRNA-329-5p with IncARAT (Fig. 5D). miRNA-329-5p expression was observed in the infiltrating macrophages following injury (Supporting Figure S4C).

To further verify the combination of miRNA-329-5p with IncARAT, the recombinant plasmids of pGL3IncARAT-wt and pGL3-IncARAT-Mut were constructed and co-transfected with miRNA-329-5p into HEK293 cells, and then the luciferase activity was assessed (Fig. 5E). Figure 5F showed that miRNA-329-5p markedly repressed the luciferase activity of pGL3-IncARAT-wt compared with miR-control, whereas the IncARAT mutation in miRNA-329-5p binding site destroyed the inhibitory effect. The direct binding of miRNA-329-5p to IncARAT was further verified using RIP assay. RIP with anti-Ago2 antibody showed that miRNA-329-5p and IncARAT were enriched in control cells (Fig. 5G). Especially, miRNA-329-5p inhibition markedly reduced the enrichment of IncARAT and miRNA-329-5p in Ago2 precipitates (Fig. $5 \mathrm{H}$ and I). The results from qPCR showed that forced expression of IncARAT in macrophages resulted in a decreased expression of miRNA-329-5p, whereas IncARAT knockdown upregulated the miRNA-329-5p expression (Fig. 5J). Functionally, IncARAT overexpression facilitated macrophage M2 polarization as indicated by the increased expression of IL-10, CD206 and Arg1, whereas additional treatment of miRNA-329-5p partially reversed the effect (Fig. $5 \mathrm{~K}$ and $\mathrm{L}$ ). These data demonstrate that IncARAT facilitates macrophage M2 polarization through sponging miRNA-329-5p and destroying its function.

\section{7 miRNA-329-5p regulated macrophage M2 polarization by targeting SOCS2-STAT1/6 signaling}

Based on the above results, we speculated that miRNA-329-5p inhibition might facilitate macrophage M2 polarization. Indeed, anti-miRNA-329-5p treatment repressed the expression of miRNA-329-5p and resulted in a subsequent increase of mRNA (Fig. 6A) and protein (Fig. 6B and C) level of macrophage M2 markers. We then explored the downstream signaling pathway regulated by miRNA-329-5p. The above results have identified 77 differentially expressed mRNAs (Supporting Table S2) in injured nerves, and meanwhile bioinformatics analysis predicted 2448 potential target genes of miRNA-329-5p using TargetScan (http://www.targetscan.org/vert_72/). Venn diagram analysis showed that there are 9 common genes in both sets of data (Fig. 6D). KEGG analysis from 77 differentially expressed mRNAs showed that the NF-KB and TNF signaling pathways were both mainly enriched pathway (Fig. 6E), and SOCS2, a crucial member of NF-KB and TNF pathways, was a potential target gene of miRNA-329-5p (Supporting Figure S5A). Then, recombinant plasmids of pGL3-SOCS2-3'UTR-WT or its mutant (pGL3- 
SOCS2-3'UTR-Mut) were constructed and co-transfected with miRNA-329-5p (Fig. 6F). The results from luciferase reporter assay showed that miRNA-329-5p significantly inhibited the luciferase expression of SOCS2-3'UTR-LUC, whereas the mutation of 4 nucleotides in the 3'UTR of SOCS2 caused the complete abrogation of the suppressive effect (Fig. 6G). miRNA-329-5p overexpression in BMDM also repressed the protein expression of SOCS2 (Fig. 6H and I). Functionally, miRNA-329-5p inhibition facilitated macrophage M2 polarization, whereas additional inhibition of SOCS2 significantly destroyed the effect (Fig. 6J), indicating the important role of SOCS2 in mediating miRNA-329-5p-inducing M2 polarization. Given the role of STAT signalling in macrophage polarization and the correlation of SOCS with STAT signalling (38), we next assessed whether SOCS2 overexpression results in the dysregulation of STAT signalling. Figure 6K-N showed that SOCS2 overexpression repressed STAT1 signalling and activated STAT6 signalling, but did affect STAT3 signalling.

\subsection{The role of IncARAT/miRNA-329-5p/SOCS2 axis in regulating macrophage $\mathrm{M} 2$ polarization}

The interaction among IncARAT, miRNA-329-5p and SOCS2, and its role in regulating macrophage polarization was then investigated. Figure 7A-C showed that IncARAT overexpression upregulated the mRNA and protein level of SOCS2 in macrophages, whereas additional treatment of miRNA-329-5p partially repressed the effect (Supporting Figure S5B). Moreover, IncARAT knockdown downregulated the mRNA and protein level of SOCS2, whereas additional repression of miRNA-329-5p significantly weakened the effect (Fig. 7D-F). To determine whether IncARAT/miRNA-329-5p/SOCS2 axis regulated macrophage M2 polarization, macrophages were co-transfected with IncARAT with or without siSOCS2 (Supporting Figure S5C). LncARAT facilitated macrophage M2 polarization as evidenced by increased expression of IL-10, Arg1 and CD206 at mRNA (Fig. 7G, Supporting Figure S5D) and protein level (Fig. 7H and I), whereas SOCS2 inhibition partially repressed the effect. Based on these data, we confirmed that IncARAT acted as a ceRNA to facilitate macrophage M2 polarization via miRNA-329-5p/SOCS2 axis. Furthermore, IncARAT overexpression repressed STAT1 signaling activation and promoted STAT6 signaling activation, whereas additional repression of SOCS2 significantly weakened the effect (Fig. 7JL).

\subsection{IncARAT-regulated macrophages function facilitated axonal regeneration and functional recovery}

Finally, we investigated whether macrophages activities regulated by IncARAT are critical to nerve regeneration. To this end, an in vivo macrophage-exhausted model was established by injecting clodronate in the presence of Lv-IncARAT or Lv-shlncARAT and then damaged the sciatic nerves after 4 days. As shown in Supporting Figure S6A, although IncARAT overexpression promoted macrophages infiltration into injured nerves, clodronate treatment depleted markedly macrophages in injured nerves. Consequently, the function of IncARAT on accelerating early demyelination was also damaged by clodronate (Supporting Figure S6B and C). The sciatic functional index was higher in IncARAT- 
overexpressed rats than in control rats, whereas the sciatic functional index was decreased similarly in control rats and IncARAT-overexpressed rats following clodronate treatment (Supporting Figure S6D), suggesting that the role of IncARAT in axonal regeneration and functional recovery was abolished due to macrophages exhaustion. To further confirm the macrophage M2 polarization promotes axonal regeneration, the damaged sciatic nerves (DNS) after 4 days was treated by M1-marophage or M2macrophage, and nerve growth factors and axonogenesis-related gene was assessed by RT-PCR, the nerve growth factors and axonogenesis-related was obviously increased by M2-macrophage treatment (Supporting Figure S6E).Taken together, these data demonstrated that upregulated IncARAT promotes axonal regeneration through recruiting macrophages and inducing macrophage $\mathrm{M} 2$ polarization (Supporting Figure S6F).

\section{Discussion}

Revealing the underlying mechanisms of SCs-macrophages interaction following $\mathrm{PNI}$ is a prerequisite to develop medicinal treatment strategies to peripheral nerve repair after injury. PNI causes extensively aberrant expression of mRNAs and non-coding RNAs (IncRNAs and miRNAs). IncRNA can function as ceRNA to regulate mRNA expression via absorbing miRNAs. In the current study, we demonstrate that (i) LncARAT expression in SCs was increased after PNI, (ii) LncARAT promoted macrophages infiltration by increasing CCL2 expression, (iii) LncARAT promoted H3K4me3 at CCL2 promoter by directly interacting with KMT2A, (iv) SCs-Exo facilitated macrophage M2 polarization through delivering IncARAT, (v) LncARAT functioned as a ceRNA and sponged miRNA-329-5p to regulate SOCS2/STAT signaling, (vi) IncARAT-regulated macrophages function facilitated axonal regeneration and functional recovery. These data reveal the important role of IncARAT in regulating the interaction of SCs with macrophages and may serve as a promising therapeutic avenue for peripheral nerve repair.

It is well known that chemokines exert a key role in macrophages recruitment and activation after PNI. Emerging studies have explored the underlying mechanisms of epigenetically activated CCL2 expression. A product of degenerated neural tissue, chitooligosaccharides, stimulates SCs to express CCL2 by repressing miR-327 in SCs (39). In a study about cancer, Chen et al., showed that IncRNA-LNMAT1 upregulates CCL2 expression via recruiting hnRNPL to the promoter of CCL2, which results in an increased H3K4me3 to facilitate CCL2 transcription and subsequent macrophages recruitment into tumor tissues (40). In the study we identified a novel highly expressed IncRNA in SCs after PNI, IncARAT. LncARAT overexpression enhanced the expression level of CCL2 in SCs in vivo and in vitro. Sequence analysis showed a distinct chromatin H3K4me3 modification on the transcription edges of the CCL2. Experimental study demonstrated that IncARAT increased CCL2 expression by recruiting KMT2A to CCL2 promoter, and resulted in an enhanced H3K4me3 that promoted CCL2 transcription. Therefore, the current results verified that SCs recruited circulating macrophages to injured nerves by the pathway of IncARAT/KMT2A/CCL2.

The main innovation of this study lie in that we revealed how SCs induces macrophage M2 polarization to facilitate axonal regeneration. Although SCs can potently induce macrophage M2 polarization (20), 
SCs could not secrete M2-associated cytokines. The underlying mechanisms that SCs regulate macrophage polarization remain poorly understood. Mounting evidences have demonstrated that exosomes exert an important role in cell-cell communication $(41,42)$, and SCs-Exo contributes to axonal regeneration by communicating with neighboring axons after $\operatorname{PNI}(2,43)$. In the study, we further revealed the role of exosomes in the interaction of SCs with macrophages. Upregulated IncARAT was packed within SCs-Exo to resist RNase-mediated degradation and then was transferred to macrophages. Subcellular localization analysis showed that IncARAT was largely located in cytoplasm in macrophages, indicating that IncARAT might act as a ceRNA, which regulate mRNA transcription via sponging miRNAs (37). Indeed, the results from RNA pull-down and RIP assay verified the direct combination of IncARAT with miRNA-329-5p. Luciferase reporter assay showed that miRNA-329-5p could specifically bind to IncARAT and repress the activity of IncARAT-LUC. RNA RIP assay with anti-Ago 2 antibody showed that miRNA-329-5p inhibition markedly reduced the enrichment of IncARAT and miRNA-329-5p in Ago2 precipitates, confirming the direct combination of IncARAT with miRNA-329-5p. Functionally, miRNA-329$5 p$ inhibition facilitated macrophage M2 polarization.

Then the target gene of miRNA-329-5p was identified and data shown that miRNA-329-5p inhibited SOCS2 expression in macrophages. IncARAT could function as an endogenous sponge to adsorb miRNA329-5p, resulting in an increased SOCS2 expression, which facilitated macrophage M2 polarization through a STAT1/6-dependent pathway, thus promoted axonal regeneration. Taken together, the present data verify that IncARAT is an important mediator of SCs-mediated macrophage infiltration and M2 polarization to support axonal regeneration, and may serve as a promising therapeutic avenue for peripheral nerve repair.

The major limitation of the present study lies in: the IncARAT-deficient $\left(\right.$ IncARAT $\left.^{-/}\right)$rats were not successfully constructed. The role of IncARAT in recruiting macrophages, inducing macrophage M2 polarization, and promoting axonal regeneration will be further verified in vivo if there is the IncARAT ${ }^{-/-}$ rats. We only used the Lv-shIncARAT to repress IncARAT expression in injured nerves.

\section{Conclusion}

The current data demonstrated that IncARAT knockdown suppressed macrophages infiltration and M2 polarization, early demyelination, and axonal regeneration.

\section{Abbreviations}

SCs

Schwann Cells

CSN

Crushed sciatic nerves

PNS

Peripheral nervous system 
SD

Sprague dawley

CMAP

Compound muscle action potential

SFI

Sciatic functional index

BMDM

Bone marrow-derived macrophages

DNS

Damaged sciatic nerves

\section{Declarations}

\section{Ethics approval and consent to participate}

The animal study was reviewed and approved by Medical Ethical Committee of Shanghai General Hospital, Shanghai Jiaotong University School of Medicine.

\section{Consent for publication}

Written informed consent for publication was obtained from all participants.

\section{Data availability}

The data supporting the findings of the article are available in and in the [GEO Dataset] at [https://www.ncbi.nlm.nih.gov/geo/], reference number [GSE149657]. More data supporting this study are available from the correspondent author.

\section{Competing interests}

The authors declare that they have no competing interests.

\section{Funding}

This work was supported by the National Natural Science Foundation of China (grant no. 81772327).

\section{Authors' contributions}

Conceptualization, GY; investigation, YL and PW; writing, JZ; supervision and funding acquisition, HL. All authors read and approved the final manuscript.

\section{Acknowledgements}

Not applicable. 


\section{References}

1. Mahar $M$, Cavalli V. Intrinsic mechanisms of neuronal axon regeneration. Nature reviews Neuroscience. 2018 Jun;19(6):323-37. PubMed PMID: 29666508. Pubmed Central PMCID: 5987780. Epub 2018/04/19. eng.

2. Lopez-Verrilli MA, Picou F, Court FA. Schwann cell-derived exosomes enhance axonal regeneration in the peripheral nervous system. Glia. 2013 Nov;61(11):1795-806. PubMed PMID: 24038411. Epub 2013/09/17. eng.

3. Scheib J, Hoke A. Advances in peripheral nerve regeneration. Nature reviews Neurology. 2013 Dec;9(12):668-76. PubMed PMID: 24217518. Epub 2013/11/13. eng.

4. Jessen KR, Mirsky R. The repair Schwann cell and its function in regenerating nerves. The Journal of physiology. 2016 Jul 1;594(13):3521-31. PubMed PMID: 26864683. Pubmed Central PMCID: 4929314. Epub 2016/02/13. eng.

5. Chen P, Piao X, Bonaldo P. Role of macrophages in Wallerian degeneration and axonal regeneration after peripheral nerve injury. Acta Neuropathol. 2015 Nov;130(5):605-18. PubMed PMID: 26419777. Epub 2015/10/01. eng.

6. Brown BN, Ratner BD, Goodman SB, Amar S, Badylak SF. Macrophage polarization: an opportunity for improved outcomes in biomaterials and regenerative medicine. Biomaterials. 2012 May;33(15):3792-802. PubMed PMID: 22386919. Pubmed Central PMCID: 3727238. Epub 2012/03/06. eng.

7. Gordon S, Taylor PR. Monocyte and macrophage heterogeneity. Nature reviews Immunology. 2005 Dec;5(12):953-64. PubMed PMID: 16322748. Epub 2005/12/03. eng.

8. Hikawa N, Takenaka T. Myelin-stimulated macrophages release neurotrophic factors for adult dorsal root ganglion neurons in culture. Cellular and molecular neurobiology. 1996 Aug;16(4):517-28. PubMed PMID: 8879753. Epub 1996/08/01. eng.

9. Zigmond RE, Echevarria FD. Macrophage biology in the peripheral nervous system after injury. Progress in neurobiology. 2019 Feb;173:102 - 21. PubMed PMID: 30579784. Pubmed Central PMCID: 6340791. Epub 2018/12/24. eng.

10. Tomlinson JE, Zygelyte E, Grenier JK, Edwards MG, Cheetham J. Temporal changes in macrophage phenotype after peripheral nerve injury. Journal of neuroinflammation. 2018 Jun 15;15(1):185. PubMed PMID: 29907154. Pubmed Central PMCID: 6003127. Epub 2018/06/17. eng.

11. Stratton JA, Holmes A, Rosin NL, Sinha S, Vohra M, Burma NE, et al. Macrophages Regulate Schwann Cell Maturation after Nerve Injury. Cell reports. 2018 Sep 4;24(10):2561-72 e6. PubMed PMID: 30184491. Epub 2018/09/06. eng.

12. Goncalves AF, Dias NG, Moransard M, Correia R, Pereira JA, Witke W, et al. Gelsolin is required for macrophage recruitment during remyelination of the peripheral nervous system. Glia. $2010 \mathrm{Apr}$ 15;58(6):706-15. PubMed PMID: 20014276. Epub 2009/12/17. eng. 
13. Allard DE, Wang Y, Li JJ, Conley B, Xu EW, Sailer D, et al. Schwann cell-derived periostin promotes autoimmune peripheral polyneuropathy via macrophage recruitment. The Journal of clinical investigation. 2018 Oct 1;128(10):4727-41. PubMed PMID: 30222134. Pubmed Central PMCID: 6159985. Epub 2018/09/18. eng.

14. Martini R, Fischer S, Lopez-Vales R, David S. Interactions between Schwann cells and macrophages in injury and inherited demyelinating disease. Glia. 2008 Nov 1;56(14):1566-77. PubMed PMID: 18803324. Epub 2008/09/23. eng.

15. Karanth S, Yang G, Yeh J, Richardson PM. Nature of signals that initiate the immune response during Wallerian degeneration of peripheral nerves. Exp Neurol. 2006 Nov;202(1):161-6. PubMed PMID: 16828744. Epub 2006/07/11. eng.

16. George A, Schmidt C, Weishaupt A, Toyka KV, Sommer C. Serial determination of tumor necrosis factor-alpha content in rat sciatic nerve after chronic constriction injury. Experimental neurology. 1999 Nov;160(1):124-32. PubMed PMID: 10630197. Epub 2000/01/12. eng.

17. Shamash S, Reichert F, Rotshenker S. The cytokine network of Wallerian degeneration: tumor necrosis factor-alpha, interleukin-1alpha, and interleukin-1beta. The Journal of neuroscience: the official journal of the Society for Neuroscience. 2002 Apr 15;22(8):3052-60. PubMed PMID: 11943808. Pubmed Central PMCID: 6757534. Epub 2002/04/12. eng.

18. Zhan C, Ma CB, Yuan HM, Cao BY, Zhu JJ. Macrophage-derived microvesicles promote proliferation and migration of Schwann cell on peripheral nerve repair. Biochemical and biophysical research communications. Dec. 2015;4-11(1-2):343-8. 468(. PubMed PMID: 26499078. Epub 2015/10/27. eng.

19. Yoshiba N, Edanami N, Ohkura N, Maekawa T, Takahashi N, Tohma A, et al. M2 Phenotype Macrophages Colocalize with Schwann Cells in Human Dental Pulp. Journal of dental research. 2020 Jan 8:22034519894957. PubMed PMID: 31913775. Epub 2020/01/09. eng.

20. Stratton JA, Shah PT, Kumar R, Stykel MG, Shapira Y, Grochmal J, et al. The immunomodulatory properties of adult skin-derived precursor Schwann cells: implications for peripheral nerve injury therapy. The European journal of neuroscience. 2016 Feb;43(3):365-75. PubMed PMID: 26121489. Epub 2015/06/30. eng.

21. Lopez-Leal R, Diaz-Viraque F, Catalan RJ, Saquel C, Enright A, Iraola G, et al. Schwann cell reprogramming into repair cells increases miRNA-21 expression in exosomes promoting axonal growth. Journal of cell science. 2020 Jun 15;133(12). PubMed PMID: 32409566. Epub 2020/05/16. eng.

22. Yao C, Chen Y, Wang J, Qian T, Feng W, Mao S, et al. LncRNA BC088259 promotes Schwann cell migration through Vimentin following peripheral nerve injury. Glia. 2020 Mar;68(3):670-9. PubMed PMID: 31721324. Epub 2019/11/14. eng.

23. Wang W, Su Y, Tang S, Li H, Xie W, Chen J, et al. Identification of noncoding RNA expression profiles and regulatory interaction networks following traumatic spinal cord injury by sequence analysis. 
Aging. 2019 Apr 18;11(8):2352-68. PubMed PMID: 30998503. Pubmed Central PMCID: 6520015. Epub 2019/04/19. eng.

24. Dadon-Nachum M, Ben-Zur T, Srugo I, Shamir HM, Melamed E, Yaffe D, et al. Therapeutic effect of myogenic cells modified to express neurotrophic factors in a rat model of sciatic nerve injury. J Stem Cells Regen Med. 2012;8(1):21-7. PubMed PMID: 24693189. Pubmed Central PMCID: PMC3908299.

25. Mao S, Zhang S, Zhou S, Huang T, Feng W, Gu X, et al. A Schwann cell-enriched circular RNA circAnkib1 regulates Schwann cell proliferation following peripheral nerve injury. FASEB journal: official publication of the Federation of American Societies for Experimental Biology. 2019 Nov;33(11):12409-24. PubMed PMID: 31415184. Pubmed Central PMCID: 6902728. Epub 2019/08/16. eng.

26. Wang X, Pan M, Wen J, Tang Y, Hamilton AD, Li Y, et al. A novel artificial nerve graft for repairing longdistance sciatic nerve defects: a self-assembling peptide nanofiber scaffold-containing poly(lacticco-glycolic acid) conduit. Neural Regen Res. 2014 Dec 15;9(24):2132-41. PubMed PMID: 25657734. Pubmed Central PMCID: PMC4316446. Epub 2015/02/07.

27. Navarro $X$. Functional evaluation of peripheral nerve regeneration and target reinnervation in animal models: a critical overview. Eur J Neurosci. 2016 Feb;43(3):271-86. PubMed PMID: 26228942. Epub 2015/08/01.

28. Campana WM, Hiraiwa M, O'Brien JS. Prosaptide activates the MAPK pathway by a G-proteindependent mechanism essential for enhanced sulfatide synthesis by Schwann cells. FASEB J. 1998 Mar;12(3):307-14. PubMed PMID: 9506474.

29. Zhang H, Li Y, de Carvalho-Barbosa M, Kavelaars A, Heijnen CJ, Albrecht PJ, et al. Dorsal Root Ganglion Infiltration by Macrophages Contributes to Paclitaxel Chemotherapy-Induced Peripheral Neuropathy. J Pain. 2016 Jul;17(7):775-86. PubMed PMID: 26979998. Pubmed Central PMCID: PMC4939513. Epub 2016/03/17.

30. Lindborg JA, Mack M, Zigmond RE. Neutrophils Are Critical for Myelin Removal in a Peripheral Nerve Injury Model of Wallerian Degeneration. The Journal of neuroscience: the official journal of the Society for Neuroscience. 2017 Oct 25;37(43):10258-77. PubMed PMID: 28912156. Pubmed Central PMCID: 5656991.

31. Xia M, Liu J, Liu S, Chen K, Lin H, Jiang M, et al. Ash1I and Inc-Smad3 coordinate Smad3 locus accessibility to modulate iTreg polarization and T cell autoimmunity. Nature communications. 2017 Jun 9;8:15818. PubMed PMID: 28598443. Pubmed Central PMCID: 5472765.

32. Malek R, Gajula RP, Williams RD, Nghiem B, Simons BW, Nugent K, et al. TWIST1-WDR5-Hottip Regulates Hoxa9 Chromatin to Facilitate Prostate Cancer Metastasis. Cancer research. 2017 Jun 15;77(12):3181-93. PubMed PMID: 28484075. Pubmed Central PMCID: 5489316.

33. Grote P, Wittler L, Hendrix D, Koch F, Wahrisch S, Beisaw A, et al. The tissue-specific IncRNA Fendrr is an essential regulator of heart and body wall development in the mouse. Developmental cell. 2013 Jan 28;24(2):206 - 14. PubMed PMID: 23369715. Pubmed Central PMCID: 4149175. 
34. Wagner KW, Alam H, Dhar SS, Giri U, Li N, Wei Y, et al. KDM2A promotes lung tumorigenesis by epigenetically enhancing ERK1/2 signaling. The Journal of clinical investigation. 2013 Dec;123(12):5231-46. PubMed PMID: 24200691. Pubmed Central PMCID: 3859406. Epub 2013/11/10. eng.

35. Trajkovic K, Hsu C, Chiantia S, Rajendran L, Wenzel D, Wieland F, et al. Ceramide triggers budding of exosome vesicles into multivesicular endosomes. Science. 2008 Feb 29;319(5867):1244-7. PubMed PMID: 18309083. Epub 2008/03/01. eng.

36. Takeuchi T, Suzuki M, Fujikake N, Popiel HA, Kikuchi H, Futaki S, et al. Intercellular chaperone transmission via exosomes contributes to maintenance of protein homeostasis at the organismal level. Proceedings of the National Academy of Sciences of the United States of America. 2015 May 12;112(19):E2497-506. PubMed PMID: 25918398. Pubmed Central PMCID: 4434695. Epub 2015/04/29. eng.

37. Thomson DW, Dinger ME. Endogenous microRNA sponges: evidence and controversy. Nature reviews Genetics. 2016 May;17(5):272-83. PubMed PMID: 27040487. Epub 2016/04/05. eng.

38. Spence S, Fitzsimons A, Boyd CR, Kessler J, Fitzgerald D, Elliott J, et al. Suppressors of cytokine signaling 2 and 3 diametrically control macrophage polarization. Immunity. 2013 Jan 24;38(1):6678. PubMed PMID: 23177319. Epub 2012/11/28. eng.

39. Zhao Y, Wang Y, Gong J, Yang L, Niu C, Ni X, et al. Chitosan degradation products facilitate peripheral nerve regeneration by improving macrophage-constructed microenvironments. Biomaterials. 2017 Jul;134:64-77. PubMed PMID: 28456077. Epub 2017/04/30. eng.

40. Chen $\mathrm{C}, \mathrm{He}$ W, Huang J, Wang B, Li H, Cai Q, et al. LNMAT1 promotes lymphatic metastasis of bladder cancer via CCL2 dependent macrophage recruitment. Nature communications. 2018 Sep 20;9(1):3826. PubMed PMID: 30237493. Pubmed Central PMCID: 6148066. Epub 2018/09/22. eng.

41. Simons M, Raposo G. Exosomes-vesicular carriers for intercellular communication. Current opinion in cell biology. 2009 Aug;21(4):575-81. PubMed PMID: 19442504. Epub 2009/05/16. eng.

42. Denzer K, Kleijmeer MJ, Heijnen HF, Stoorvogel W, Geuze HJ. Exosome: from internal vesicle of the multivesicular body to intercellular signaling device. Journal of cell science. $2000 \mathrm{Oct} ; 113 \mathrm{Pt}$ 19:3365-74. PubMed PMID: 10984428. Epub 2000/09/14. eng.

43. Lopez-Leal R, Court FA. Schwann Cell Exosomes Mediate Neuron-Glia Communication and Enhance Axonal Regeneration. Cellular and molecular neurobiology. 2016 Apr;36(3):429 - 36. PubMed PMID: 26993502. Epub 2016/03/20. eng.

\section{Figures}


A
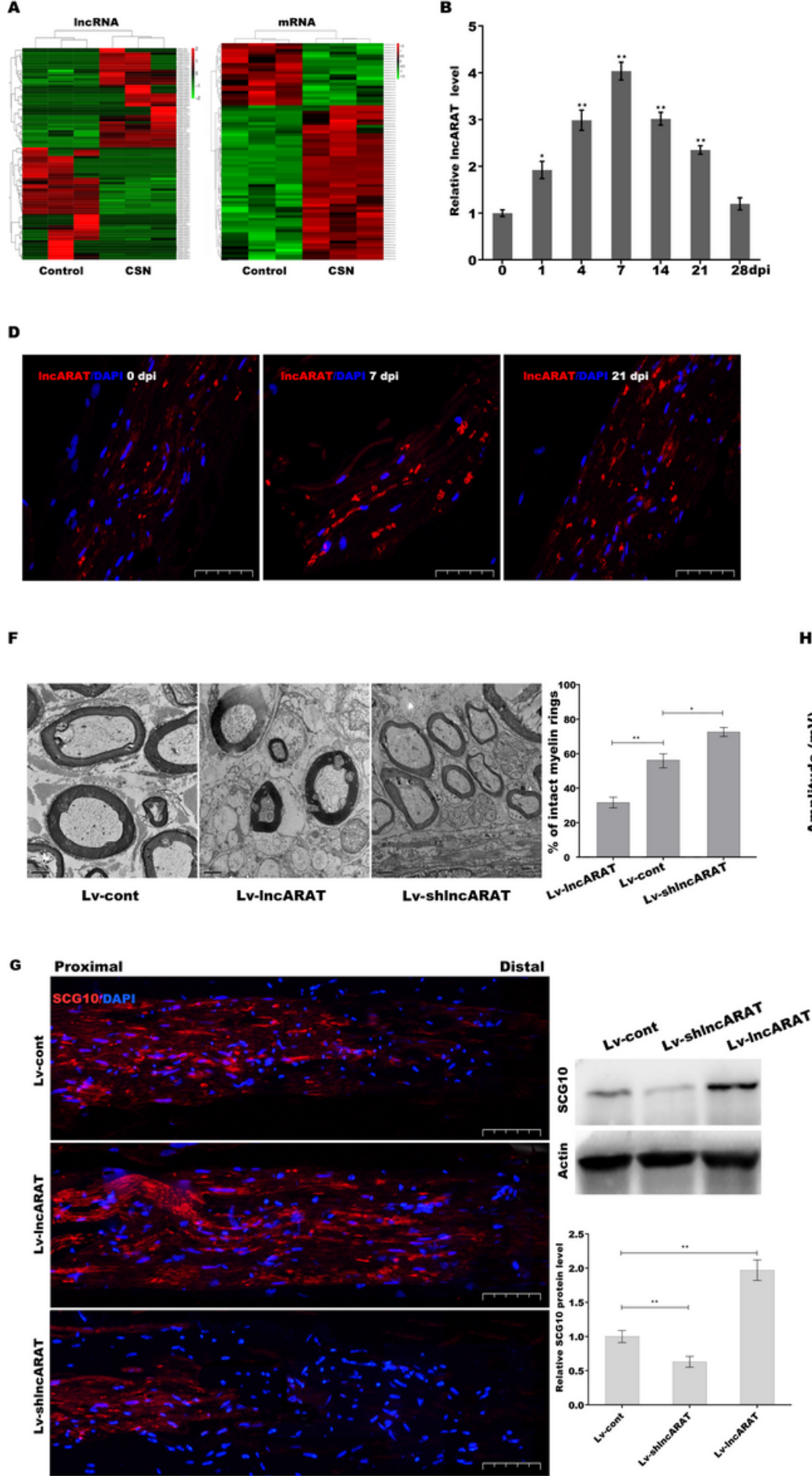

c
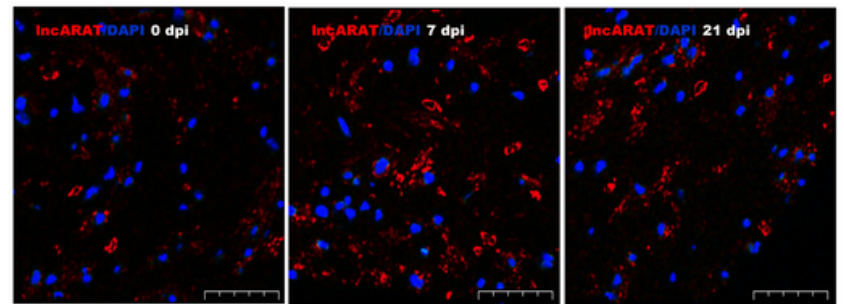

E

$\mathbf{s} 100 \beta$
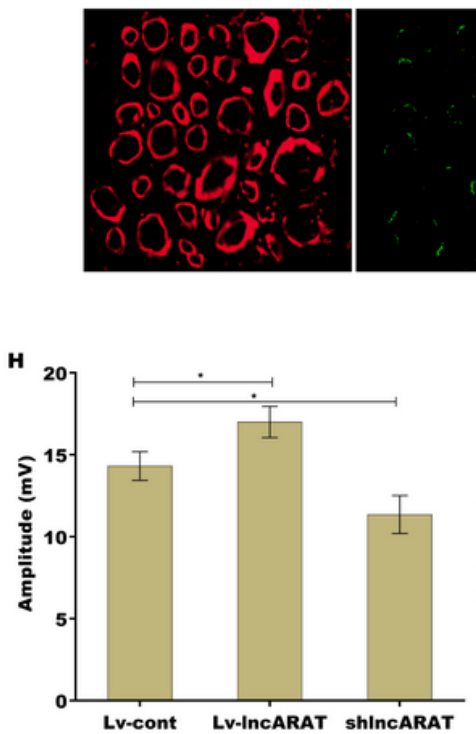

InCARAT
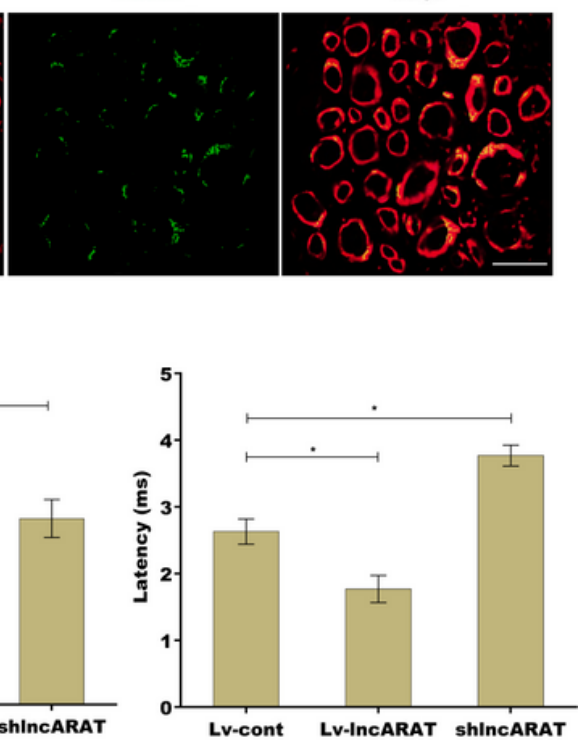

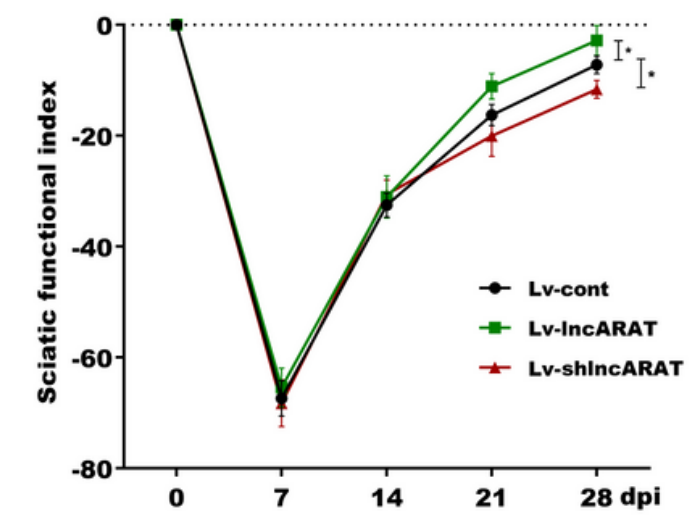

Figure 1

LncRNA expression profile in crushed sciatic nerves (A) Heat map of differentially expressed IncRNAs and mRNAs between crushed sciatic nerves $(n=3)$ and normal control $(n=3)$ at 4 dpi. (B) qPCR analysis of the IncARAT level in crushed sciatic nerves $(n=5)$ at different time points post-injury $(0,1,4,7,14,21,28 \mathrm{dpi})$. (C and D) The IncARAT level was determined in cross sections of sciatic nerves (C) and longitudinal sections of sciatic nerves (D) at different time points post-injury $(0,7,21 \mathrm{dpi})$ using immunofluorescence

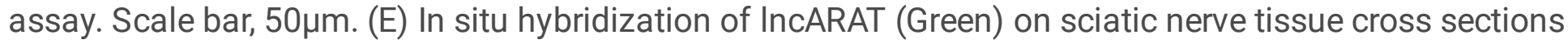




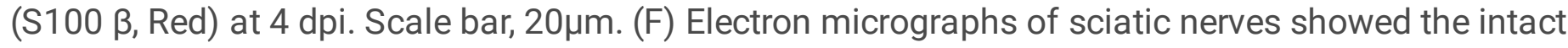
myelin after IncARAT overexpression or knockdown, Scale bar, $2 \mu \mathrm{m}$. (G) Immunofluorescence and western blot analysis of SCG10 in injured nerves after IncARAT overexpression or knockdown at $3 \mathrm{dpi}$.

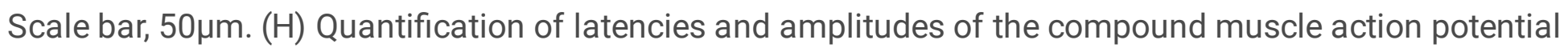
(CMAP) after IncARAT overexpression or knockdown $(n=5)$. (I) Plot of the sciatic functional index (SFI) obtained with walking track analysis after sciatic nerve crush in IncARAT overexpression or knockdown rats ( $n=5$ per group). ${ }^{*} p<0.05$. ${ }^{*} p<0.01$.
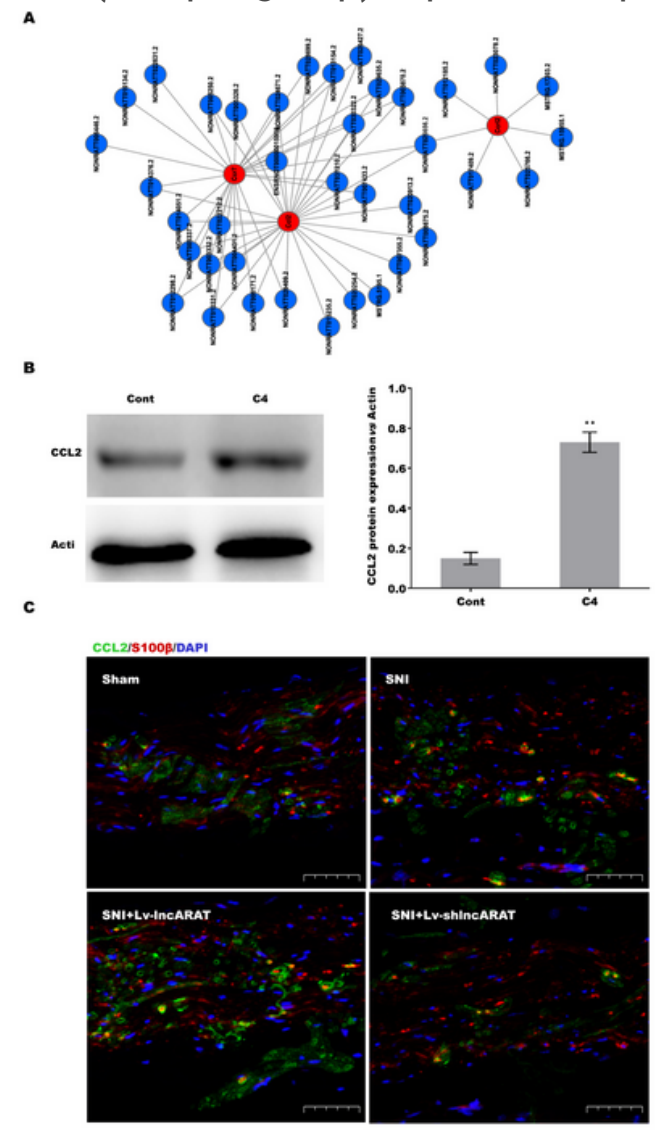

D
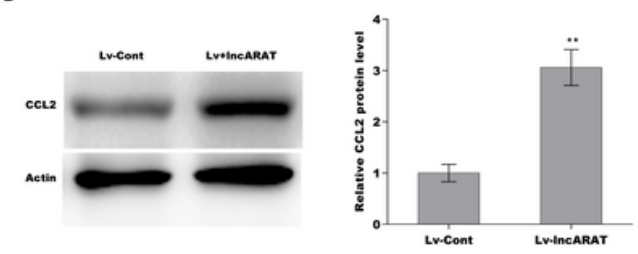
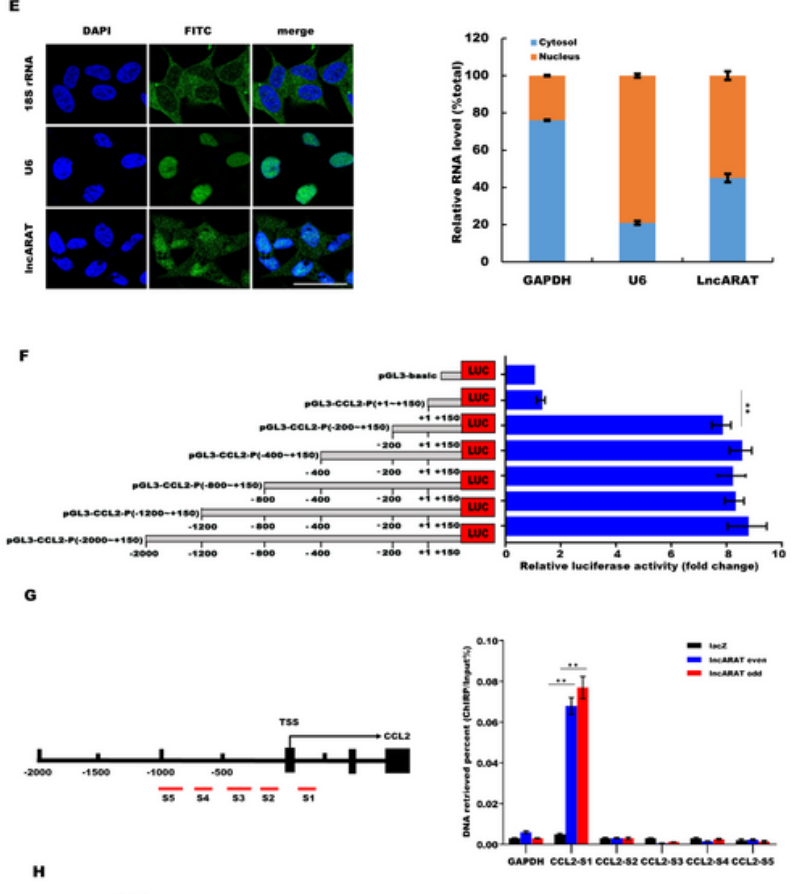

H

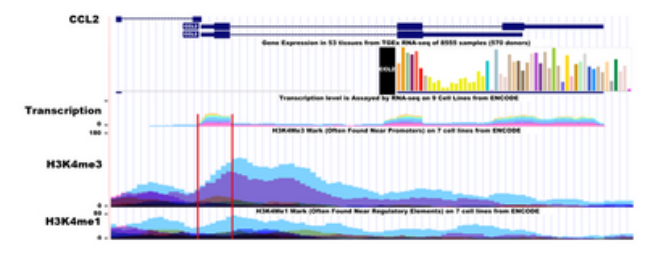

'

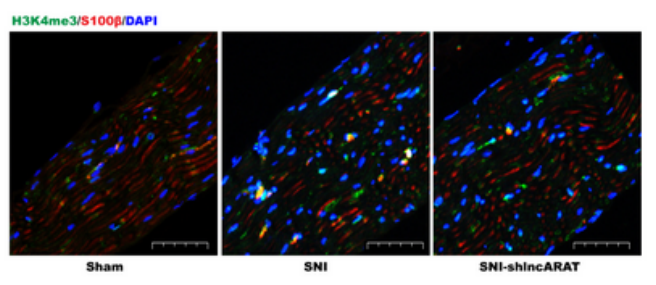

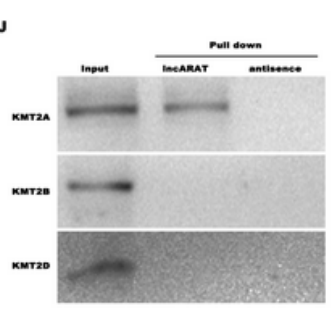

K
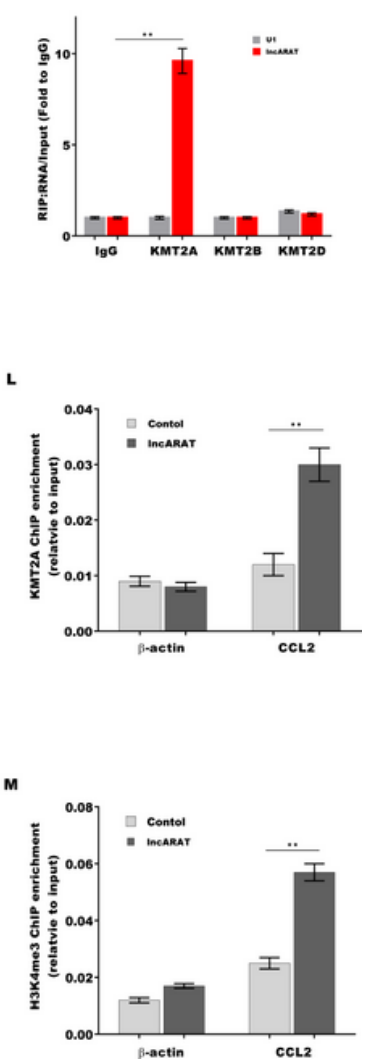

Figure 2

LncARAT upregulated CCL2 expression in SCs and promoted H3K4me3 at CCL2 promoter by interacting with KMT2A (A) A coexpression network of IncRNAs in Profile 7 with correlated target genes. IncRNAs and coding mRNAs are presented as blue and red circles, respectively. The solid line between IncRNAs and coding mRNAs indicates positive correlation in expression. (B) Western blot analysis of the CCL2 expression in injured nerves at $4 \mathrm{dpi}$. (C) Immunofluorescence assay of the CCL2 (green) and S100 $\beta$ (red) in injured nerves after IncARAT overexpression or knockdown. Scale bar, 50 $\mathrm{mm}$. (D) Western blot analysis of the CCL2 expression in primary SCs after LncARAT overexpression. (E) Left panel, RNA-FISH assay for 
IncARAT, U6, and 18S rRNA using FITC-labelled probes. Scale bar, $20 \mu \mathrm{m}$. Right panel, the cytosol and nucleus fractions were separated, and the RNA levels of IncARAT, U6, and 18S were assessed using qPCR. U6 was served as a positive control for nuclear RNA, 18S rRNA was served as a positive control for cytoplasm RNA ( $n=3)$. ( $F$ ) Sequential deletions of the CCL2 promoter linked to Renilla luciferase were constructed and the transcriptional activity was assessed. (G) Left panel, schematic presentation of the potential IncARAT binding sites in the CCL2 promoter. Right panel, ChIRP assessment of IncARATassociated chromatin in SCs. $(\mathrm{H})$ Genomic neighbourhood of CCL2. Genome browser tracks from the UCSC genome browser showing H3K4me3 occupancy near CCL2. (I) Immunofluorescence assay of the H3K4me3 level (green) and S100ß (red) in injured nerves after IncARAT overexpression or knockdown.

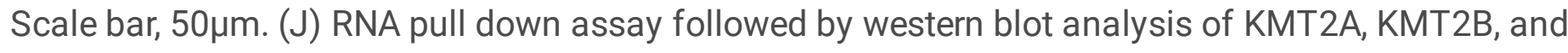
KMT2D. (K) RIP assay using the anti-KMT2A, KMT2B, or KMT2D antibody in SCs. U1 was used as the negative control. ChIP analyses of KMT2A $(L)$ and H3K4me3 $(M)$ on the regulatory regions of CCL2 genes in macrophages treated with Lv-IncARAT. ${ }^{*} p<0.05 .{ }^{*} p<0.01$. 

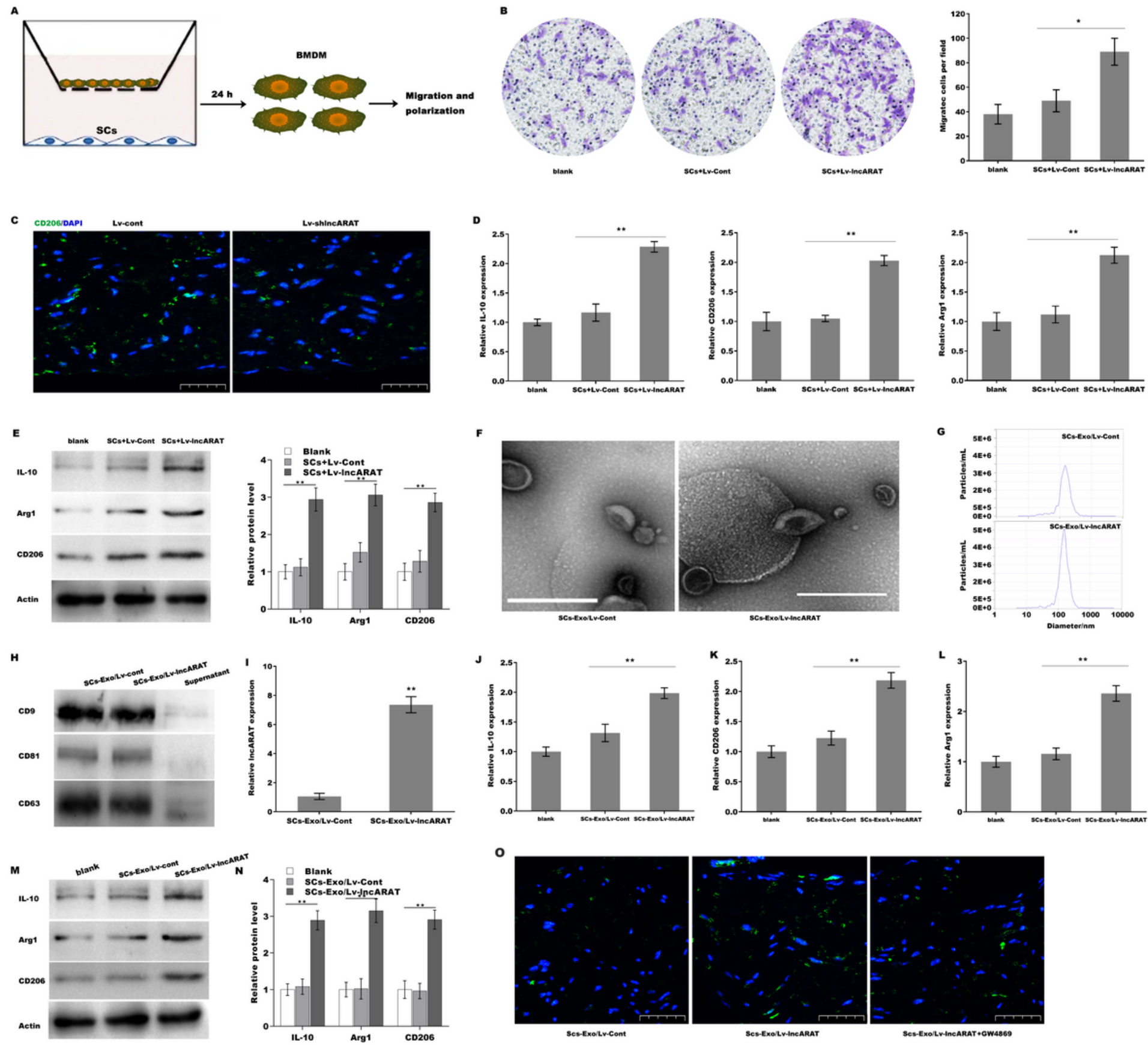

\section{Figure 3}

SCs-Exo promoted macrophage M2 polarization (A) Schematic presentation of an in vitro co-culture system for assessing the role of SCs in regulating macrophages function. (B) Transwell migration assay of macrophages co-cultured with SCs with or without IncARAT overexpression. (C) Immunofluorescence

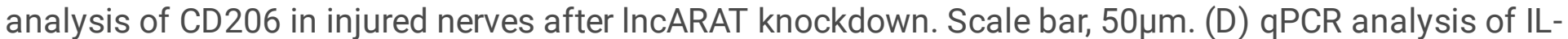
10, CD206, and Arg1 expression in macrophages co-cultured with SCs with or without IncARAT overexpression. (E) Western blot analysis of IL-10, CD206, and Arg1 expression in macrophages cocultured with SCs in the presence or absence of Lv-IncARAT. (F) Transmission electron microscopy images of exosomes isolated from SCs-Exo/Lv-cont and SCs-Exo/Lv-IncARAT. Scale bar, $500 \mathrm{~nm}$. (G) Nanoparticle tracking analysis of SCs-Exo/Lv-cont and SCs-Exo/Lv-IncARAT. (H) Western blot analysis of 
exosomal markers (CD9, CD81, and CD63) in SCs-Exo/Lv-cont and SCs-Exo/Lv-IncARAT. (I) qPCR analysis of IncARAT in SCs-Exo/cont and SCs-Exo/Lv-IncARAT. (J-L) qPCR analysis of IL-10, CD206, and Arg1 expression in macrophages after treatment with SCs-Exo/Lv-cont or SCs-Exo/Lv-IncARAT. (M) Western blot analysis of IL-10, CD206, and Arg1 expression in macrophages after treatment with SCs-Exo/Lv-cont or SCs-Exo/Lv-IncARAT. (N) Quantification of western blot bands showed in (M). (O) Immunofluorescence analysis of CD206 in macrophages after treatment with SCs-Exo/Lv-IncARAT in the presence or absence

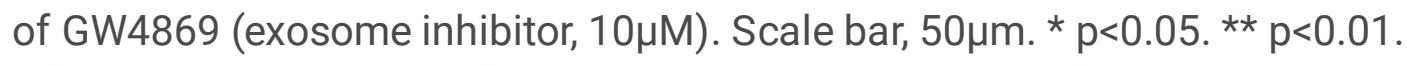
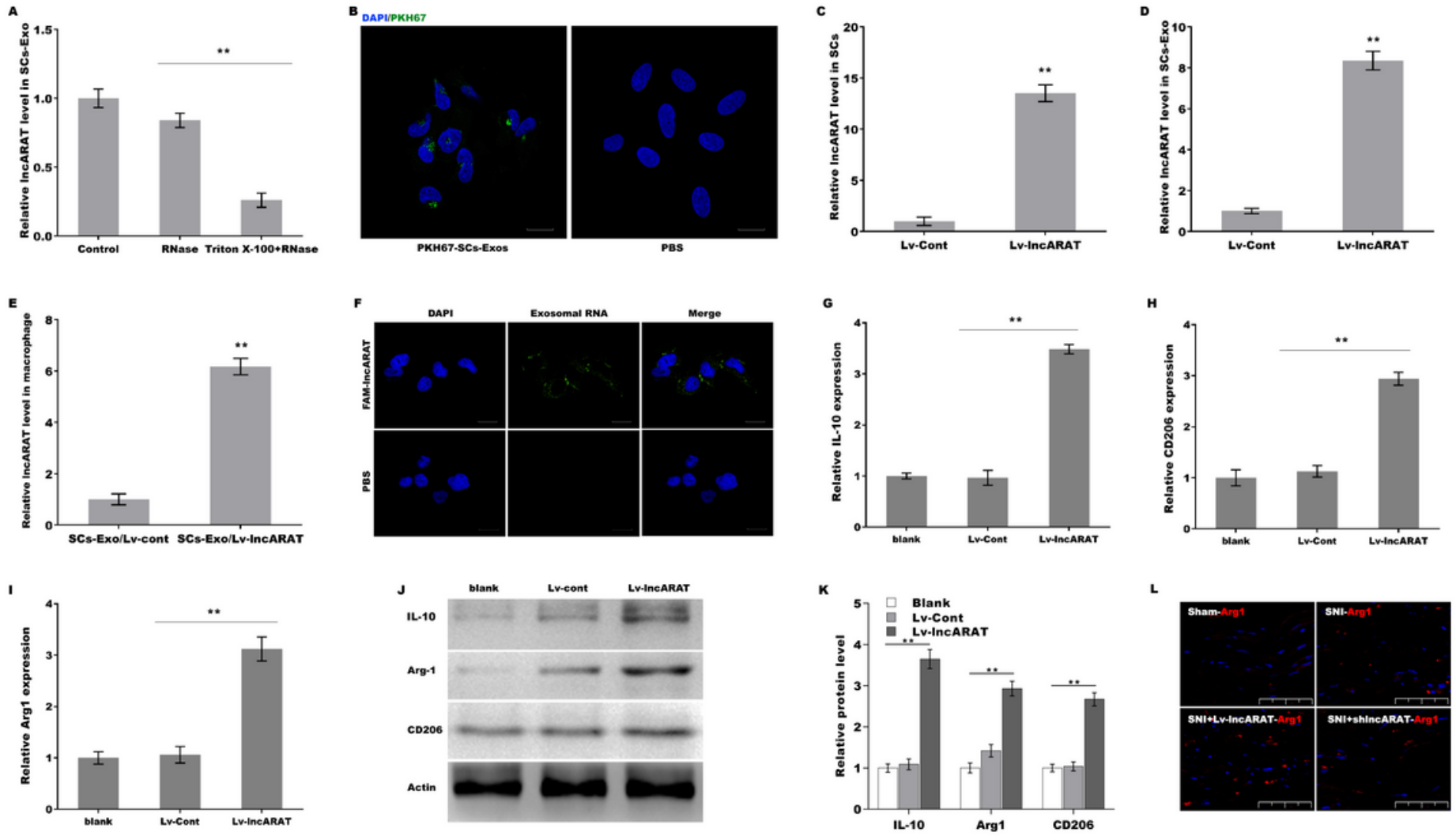

\section{Figure 4}

IncARAT was transferred from SCs to macrophages via SCs-Exo (A) qPCR analysis of IncARAT expression in SCs-Exo after RNase treatment in the presence or absence of Triton X-100. (B) Fluorescence microscopy analysis of macrophages treated with PKH67 (green)-labeled SCs-Exo, PBS served as a negative control. Scale bar, $10 \mu \mathrm{m}$. qPCR analysis of IncARAT expression in SCs (C) and SCs-Exo (D) after IncARAT overexpression. (E) qPCR analysis of IncARAT expression in macrophages treated with SCsExo/Lv-cont or SCs-Exo/Lv-IncARAT. (F) Fluorescence microscopy analysis of macrophages treated with SCs-Exo/FAM-IncARAT. Scale bar, $10 \mu \mathrm{m}$. (G-I) qPCR analysis of IL-10, CD206, and Arg1 expression in macrophages after treatment with Lv-cont or Lv-IncARAT. (J) Western blot analysis of IL-10, CD206, and Arg1 expression in macrophages after treatment with Lv-cont or Lv-IncARAT. (K) Quantification of western blot bands showed in (J). (L) Immunofluorescence assay of the Arg1 expression in cross sections of sciatic nerves from sham or rats with CSN after overexpression or knockdown of Lv-IncARAT. Scale bar, $100 \mu \mathrm{m} .{ }^{*} \mathrm{p}<0.05 . * * \mathrm{p}<0.01$. 

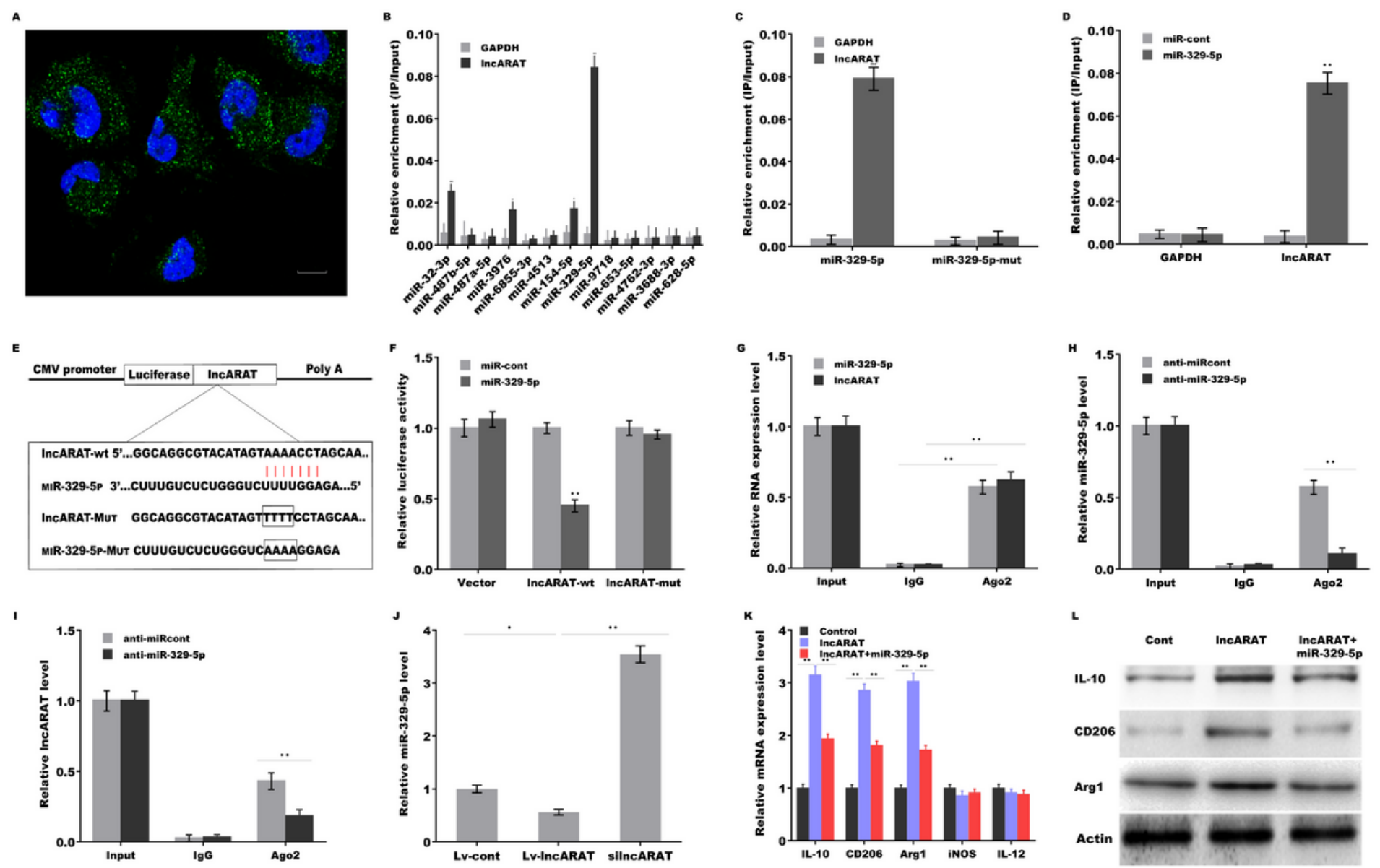

\section{Figure 5}

LncARAT sponged miRNA-329-5p to facilitate macrophage M2 polarization (A) Sub-cellular localization analysis of IncARAT in macrophages using RNA-FISH analysis. Scale bar, 10 $\mu \mathrm{m}$. (B) Biotinylated miRNAs were transfected to macrophages and then RNA pull-down assay was carried out to assess the combination of IncARAT with specific miRNA. (C) Biotinylated miRNA-329-5p mutant were transfected to macrophages and then RNA pull-down assay was carried out. (D) Biotinylated IncARAT was transfected to macrophages and then RNA pull-down assay was carried out. (E) Schematic representation of the miRNA-329-5p site in IncARAT-3'UTR. (F) Luciferase activity was assayed in HEK293 cells co-transfected with miRNA-329-5p and luciferase reporters containing IncARAT-3'UTR. (G) RIP analyses with anti-Ago2 antibody were performed to assess the enrichment of IncARAT and miRNA-329-5p in macrophages. RIP assay with anti-Ago2 antibody were performed to assess the enrichment of miRNA-329-5p $(\mathrm{H})$ and IncARAT (I) after miRNA-329-5p inhibition. (J) qPCR analysis of miRNA-329-5p expression in macrophages after IncARAT overexpression or knockdown. qPCR (K) and western blot (L) analysis of IL10, CD206, and Arg1 expression in macrophages after Lv-IncARAT overexpression in the presence or absence of miRNA-329-5p. * $p<0.05 .{ }^{* *} p<0.01$. 

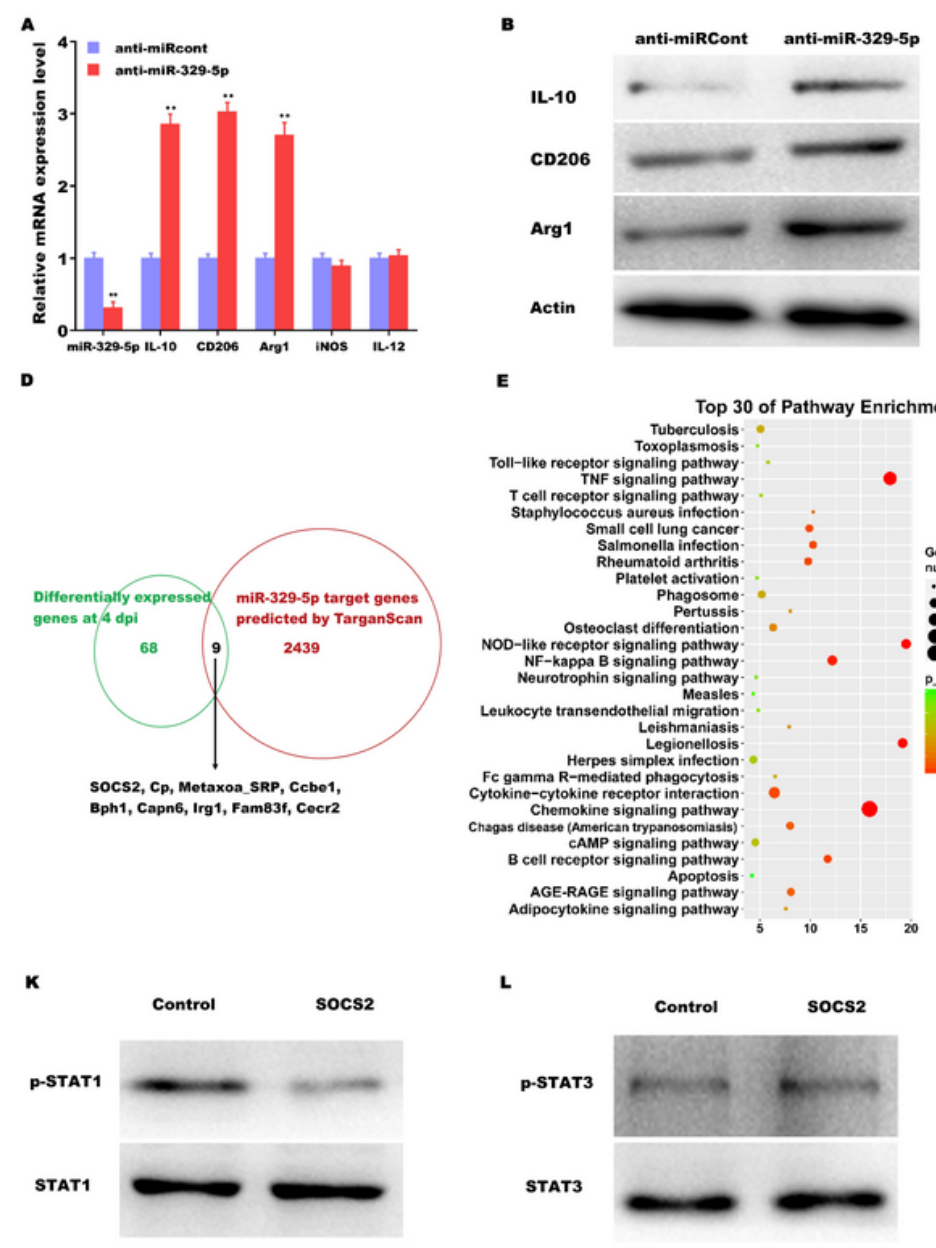
Bph1, Capn6, Irg1, Fam83i, Cecr2
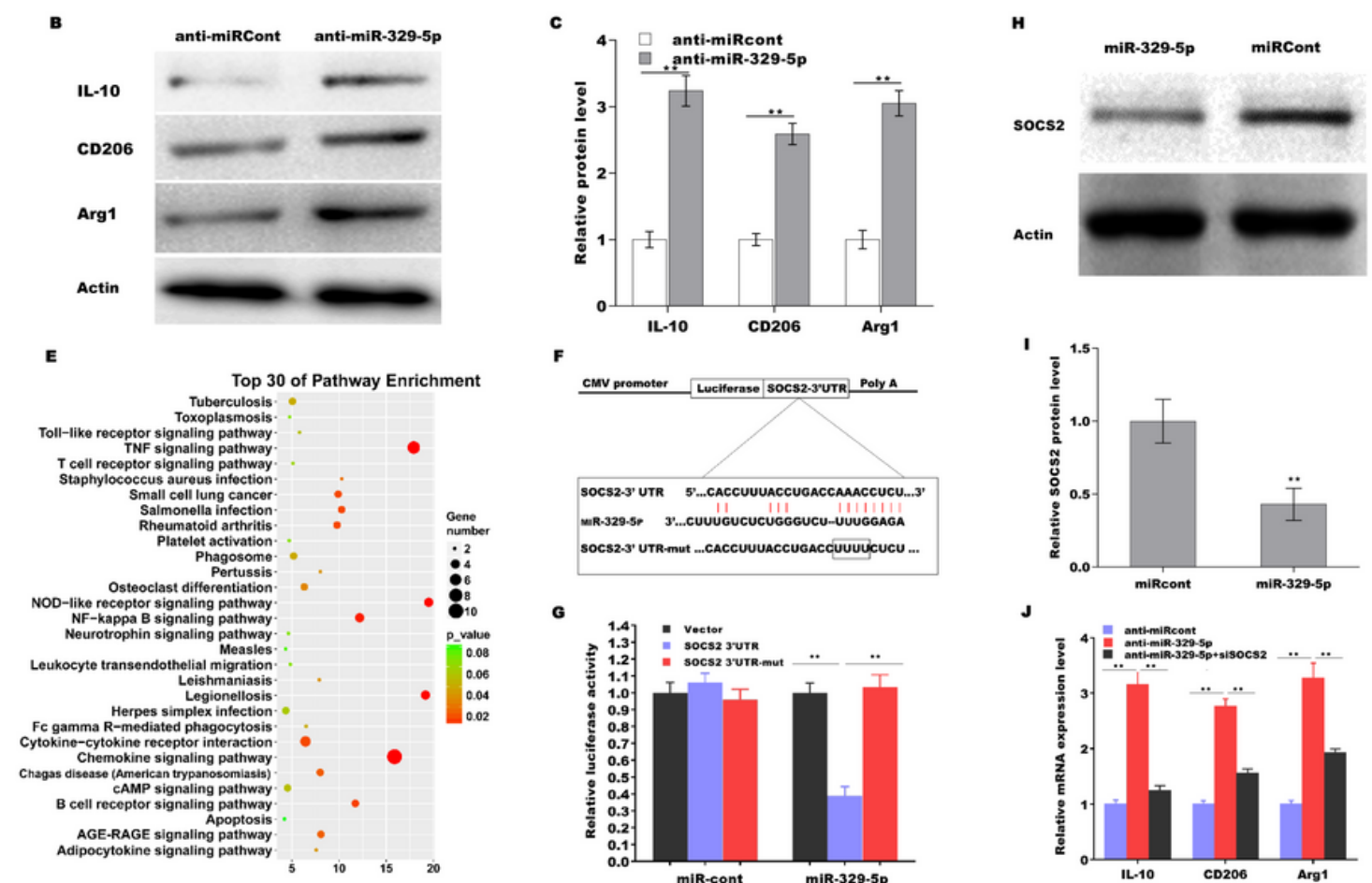

Socs2, Cp, Metaxoa_sRP, Ccbo1,

\section{西}
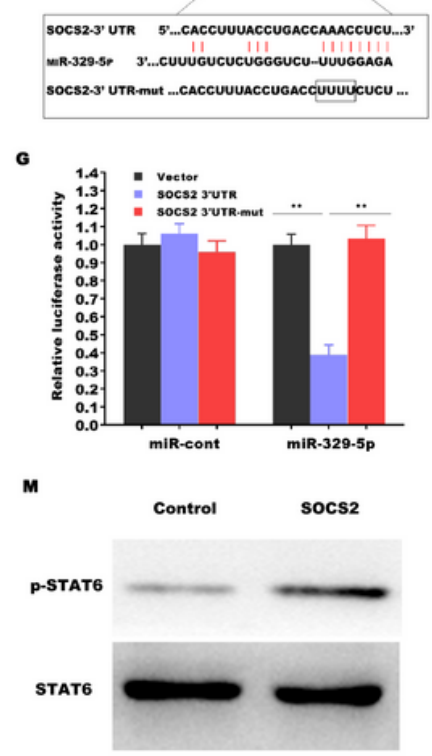

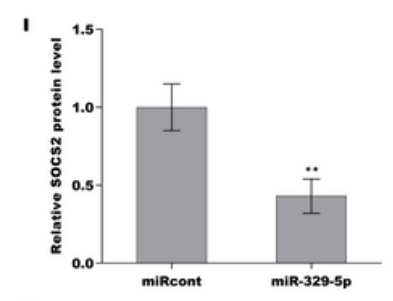

$\mathbf{J}$
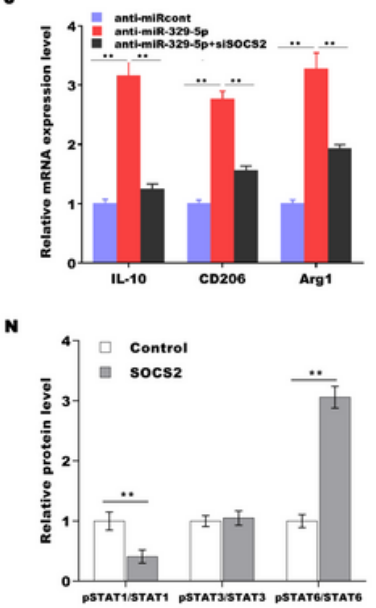

\section{Figure 6}

miRNA-329-5p regulated macrophage M2 polarization by targeting SOCS2-STAT1/ 6 signaling qPCR (A) and western blot (B and C) analysis of IL-10, CD206, and Arg1 expression in macrophages after miRNA329-5p inhibition. (D) A Venn diagram analysis of overlapping mRNAs in both sets of data. (E) KEGG analysis of 77 differentially expressed mRNAs in crushed sciatic nerves. (F) Schematic representation of the miRNA-329-5p site in SOCS2-3'UTR. (G) Luciferase activity was assayed in HEK293 cells cotransfected with miRNA-329-5p and luciferase reporters containing SOCS2-3'UTR. (H and I) Western blot analysis of SOCS2 in macrophages after miRNA-329-5p inhibition. (J) qPCR analysis of IL-10, CD206, and Arg1 expression in macrophages after miRNA-329-5p inhibition in the presence or absence of siSOCS2. (K-N) Western blot analysis of p-STAT1, p-STAT3, and p-STAT6 in macrophages after SOCS2 overexpression. ${ }^{*} p<0.05$. ${ }^{\star *} p<0.01$. 

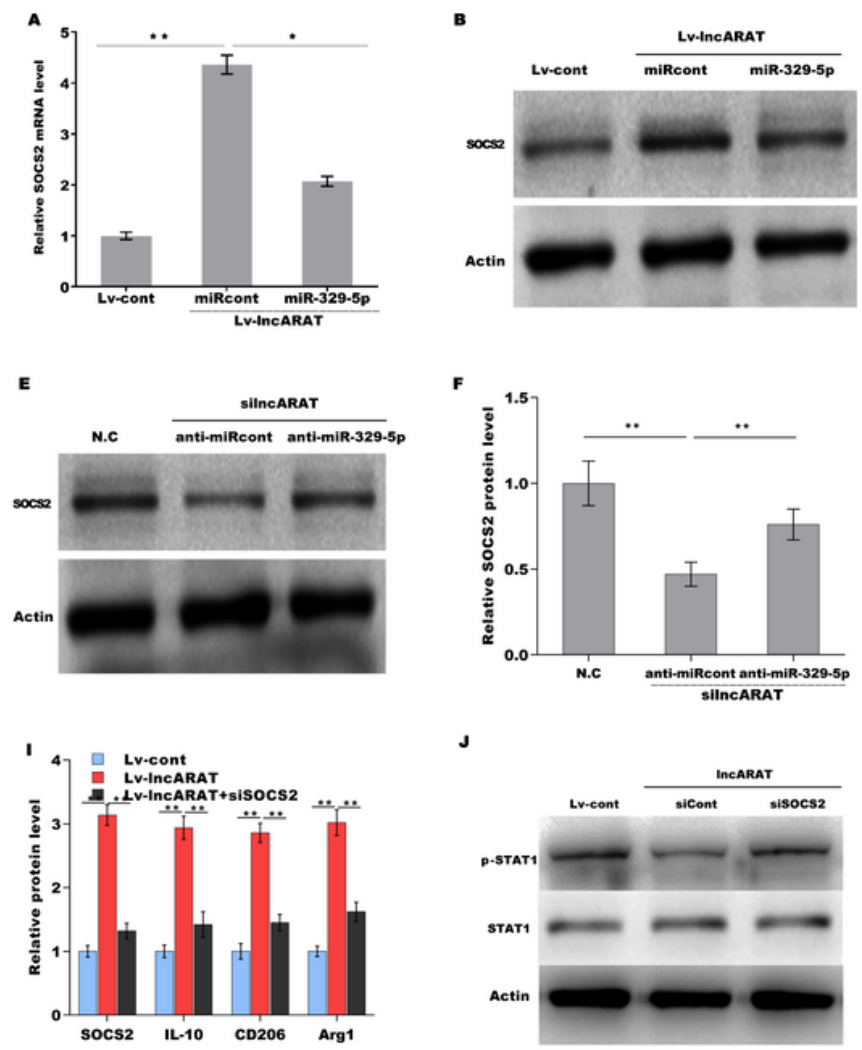
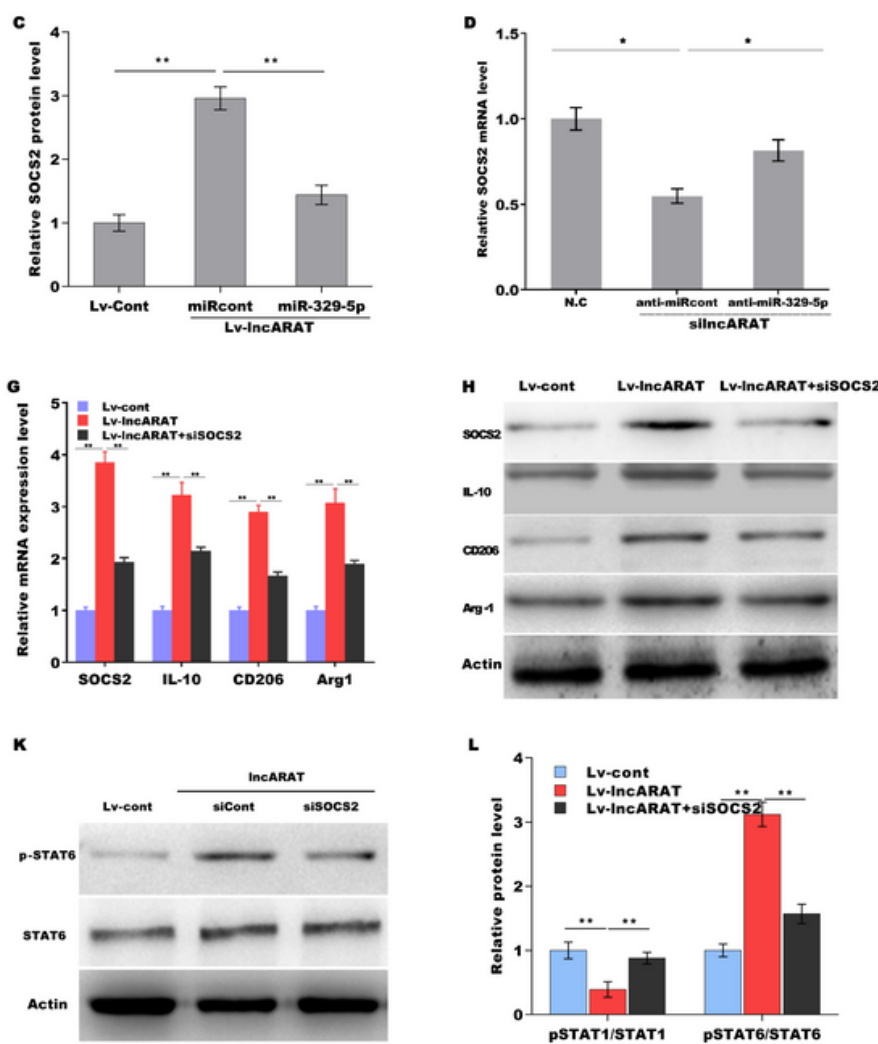

\section{Figure 7}

The role of IncARAT/miRNA-329-5p/SOCS2 axis in regulating macrophage M2 polarization qPCR (A) and western blot (B and $C$ ) analysis of SOCS2 expression in macrophages treated with IncARAT in the presence or absence of miRNA-329-5p. qPCR (D) and western blot (E and F) analysis of SOCS2 expression in macrophages treated with silncARAT in the presence or absence of miRNA-329-5p inhibitor. qPCR (G) and western blot ( $\mathrm{H}$ and $\mathrm{I}$ ) analysis of IL-10, CD206, and Arg1 expression in macrophages treated with IncARAT in the presence or absence of siSOCS2. (J-L) Western blot analysis of p-STAT1 and p-STAT6 level in macrophages treated with IncARAT in the presence or absence of siSOCS2. * $p<0.05$. ** $\mathrm{p}<0.01$.

\section{Supplementary Files}

This is a list of supplementary files associated with this preprint. Click to download.

- SupportingFigureS1.png

- SupportingFigureS2.png

- SupportingFigureS3.png

- SupportingFigureS4.png

- SupportingFigureS5.png

- SupportingFigureS6.png 
- SupportingTableS1.xlsx

- SupportingTableS2.xlsx

- SupportingTableS3.xlsx

- SupportingTableS4.xlsx

- SupportingTableS5.xlsx

- SupportingTableS6.xlsx

- SupportingTableS7.xlsx

- SupportingTableS8.docx

- SupportingTableS9.docx 